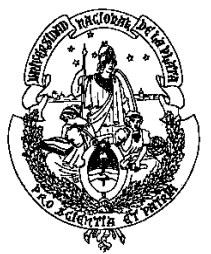

Maestría en Economía

Facultad de Ciencias Económicas

Universidad Nacional de La Plata

TESIS DE MAESTRIA

ALUMNO

Germán Reyes

TITULO

La Distribución de Spreads Ex-Post de los Países Emergentes

DIRECTOR

Martín Guzmán y Daniel Heymann (codirector)

FECHA DE DEFENSA

$12 / 2 / 2015$ 


\title{
La distribución de spreads ex-post de los países emergentes
}

\author{
Germán Reyes $^{\dagger}$
}

Tesis

Maestría en Economía

Universidad Nacional de La Plata

\author{
Directores: \\ Martín Guzmán \\ Daniel Heymann
}

Noviembre, 2015

\footnotetext{
${ }^{\dagger}$ Agradezco especialmente a mis directores Martín Guzmán y Daniel Heymann -dos auténticos outliers- por su colaboración y dedicación durante el proceso de tesis. Agradezco también a los integrantes de la Dirección Provincial de Estudios y Proyecciones Económicas (DPEPE) del Ministerio de Economía de la Provincia de Buenos Aires, del Centro de Estudios Distributivos Laborales y Sociales (CEDLAS) y de la Práctica Global de Pobreza y Equidad del Banco Mundial por promover un ambiente colaborativo que fomenta la discusión de ideas; a los participantes del Seminario de Avance de Tesis, de la XLIX Reunión Anual de la Asociación Argentina de Economía Política y del Taller Anual sobre Pobreza, Desarrollo y Globalización organizado por la Universidad Azim Premji y el Initiative for Policy Dialogue por sus comentarios y sugerencias; a los comentarios de dos referís anónimos del VII Congreso Nacional de Estudiantes de Postgrado en Economía y a Mariano Moschione, Lucas Longo y Augusto Hassel por su colaboración para conseguir los datos de Bloomberg. Errores y omisiones son de mi exclusiva responsabilidad.
} 


\title{
Resumen
}

Se calcula la distribución del spread ex-post entre el retorno de la deuda pública y el retorno de las letras del tesoro americano a diez años para una muestra de 37 países emergentes durante el periodo 1970-2012 usando datos sobre flujos agregados. El spread ex-post medio del periodo es del $0,3 \%$, lo cual evidencia que los mercados de deuda soberana efectivamente compensaron los riesgos en el periodo bajo análisis. Este resultado es robusto a analizar diversos supuestos sobre el precio final de la deuda en el mercado secundario. Los spreads ex-post medios muestran una trayectoria creciente en el tiempo. No se encuentra una relación robusta entre variables que indican percepciones sobre "tendencias pro-mercado" y los spreads ex-post. Variables que típicamente se consideran como determinantes de la rentabilidad exhiben un poder explicativo bajo de los spreads.

Clasificación JEL: F34, G15, H63

Palabras clave: Deuda soberana, spreads de retornos ex-post

\begin{abstract}
We calculate the distribution of the ex-post spread between the returns of government bonds and the return of a ten year treasury bill for a sample of 37 emerging countries during the 1970-2012 period using aggregate flows. The average spread for the period is $0.3 \%$, which shows that the sovereign debt markets effectively offset the risks. This result is robust under various assumptions about the final price of the debt in the secondary market. The mean of the ex-post spreads exhibits an upward trend over time. We cannot find a robust relationship between variables that indicate perceptions of "promarket trends" and ex-post spreads. Variables which are typically considered as determinants of returns exhibit low explanatory power of spreads.
\end{abstract}

JEL classification: F34, G15, H63

Key words: Sovereign debt, ex-post spreads of returns 


\section{Índice}

1 Introducción 4

2 Literatura relacionada $\quad 11$

3 Estimación de los spreads $\quad 13$

3.1 Fuentes de información . . . . . . . . . . . . . . . . 15

3.2 Metodología . . . . . . . . . . . . . . . . . . . 17

4 Resultados $\quad 22$

4.1 Retornos de la deuda y spreads soberanos . . . . . . . . . . . . 23

4.2 Robustez de los resultados y análisis de sensibilidad . . . . . . . . . 26

4.3 Extensiones . . . . . . . . . . . . . . . . . . . . . . . . . . . . . 29

4.4 Spreads ex-post y percepciones del mercado . . . . . . . . . . . . 32

5 Reflexiones finales $\quad 35$

$\begin{array}{ll}\text { Apéndice } & 37\end{array}$ 


\section{Introducción}

El endeudamiento es una de las principales fuentes de recursos del sector público. La deuda de los gobiernos soberanos -reinos, ciudades-estado e imperios- fue uno de los primeros activos financieros en negociarse, y en la actualidad comprende alrededor del 20\% del total de los activos financieros (Tomz y Wright, 2013). Es por esto que los contratos de deuda son fundamentales para el funcionamiento de los gobiernos y, a su vez, de la economía global. Por su parte, las crisis de deuda y los defaults soberanos son tan antiguos como el endeudamiento soberano mismo. El primer default registrado data del Siglo IV a.C., cuando diez de las trece municipalidades griegas de la Asociación Marítima del Ático cesaron los pagos de un préstamo otorgado por el Templo Delos (Wrinkler, 1933).

La característica que distingue a la deuda soberana de la deuda privada es la escasez de mecanismos para hacerla exigible. Mientras que un agente privado está siempre sujeto a una autoridad legal, no sucede lo mismo con los gobiernos soberanos. Los recursos legales a disposición de los acreedores son muy limitados en caso que el soberano incumpla con las condiciones del contrato. Más aún, a diferencia de los préstamos entre privados, los préstamos a los gobiernos no están respaldados por ninguna garantía, lo cual agrava el potencial problema de incumplimiento.

Los gobiernos cuentan con diversos instrumentos de deuda a disposición, los cuales es posible sintetizar en dos: préstamos y bonos.

Un préstamo soberano es un arreglo contractual en el cual el prestatario -en este caso, el gobierno- recibe un monto determinado de dinero en un momento dado del tiempo y a cambio se compromete pagarle al prestamista en un plazo definido bajo ciertas condiciones. Las instituciones que le proveen crédito a los gobiernos soberanos son múltiples e incluyen organismos multilaterales de crédito, tales como el Banco Mundial o el Fondo Monetario Internacional (FMI); bancos privados de inversión; y otros gobiernos soberanos, como los integrantes del Club de París.

La otra forma en que lo países se endeudan es emitiendo activos de renta fija, genéricamente conocidos con el nombre de bonos. Los bonos representan "préstamos de dinero" que reciben los soberanos por parte de los acreedores a través de su colocación en el mercado de capitales. A cambio del préstamo, el bono otorga a su 
tenedor el derecho a recibir una serie de flujos de fondos a futuro, compuestos por la amortización de capital más los intereses. A diferencia de los activos reales -los cuales proveen a su titular de servicios físicos- los bonos son títulos que confieren derechos de tipo económico sobre otro agente.

Las emisiones de bonos se venden inicialmente en el mercado primario, en donde los gobiernos soberanos se ponen en contacto por primera vez con los acreedores para obtener financiamiento. Posteriormente, dichos bonos se comercian activamente en el mercado secundario, en donde diariamente se compran y venden los bonos que fueron colocados con anterioridad en el mercado primario. La interacción entre la oferta y la demanda de este último mercado fija el precio de los bonos y, de manera implícita, su tasa de retorno. Cada bono cuenta con determinadas condiciones de emisión que determinan el perfil de rentabilidad y riesgo del activo. Dichas condiciones surgen del marco legal bajo el cual se emite el bono y están establecidas en el prospecto de emisión. Las principales características que determinan el perfil de cada bono son su unidad de denominación, la fecha de emisión y de vencimiento, la amortización o pago de capital, la tasa de interés o cupón que se aplicará sobre el capital aún no amortizado y el valor nominal.

En la práctica existe un amplio menú de instrumentos de deuda soberana con distintos perfiles de riesgo y retorno sobre el cual los inversores pueden elegir. Dado que los préstamos y los bonos comparten muchas características en común, el tratamiento de ambos instrumentos a fines analíticos será el mismo a lo largo del trabajo. Para simplificar el problema, supóngase que la decisión de inversión de un agente se reduce a elegir entre un bono emitido por un soberano "riesgoso" -el cual con cierta probabilidad no pagará sus compromisos- y un bono emitido por un gobierno "libre de riesgo" -el cual siempre paga sus deudas-, ambos en la misma denominación. El bono libre de riesgo brinda un retorno presumiblemente seguro. Sin embargo, en caso de que el soberano cumpla sus obligaciones, el bono riesgoso ofrece un retorno mayor debido a que éste refleja el hecho de que el bono tiene cierta probabilidad de no pago. Ahora bien, también es posible que el soberano riesgoso entre en default y el bono cese sus pagos, en cuyo caso el país podrá reestructurar su deuda en default y ofrecerle a los inversores un valor de recupero con el fin de poder volver a los mercados de deuda. 
En un mercado de crédito competitivo con inversores neutrales al riesgo el retorno esperado de ambos bonos es el mismo, ya que de lo contrario existirían oportunidades de obtener beneficios mediante operaciones de arbitraje. Ex-post, es posible que los retornos sean distintos. Esto se puede deber a que los inversores percibieran probabilidades de default distintas de las verdaderas o a que el valor de recupero esperado por los acreedores en caso de default fuese menor al efectivamente recibido. Si el retorno del bono riesgoso ex-post fuese mayor al del libre de riesgo, lo óptimo para los inversores hubiera sido comprar una mayor cantidad de deuda riesgosa. Al aumentar la demanda del bono se incrementa su precio y cae su tasa de retorno hasta el punto en el que su retorno es igual al del bono no riesgoso.

Los contratos de deuda soberana orientan sobre lo que ocurrirá en caso de que el gobierno no pueda pagar lo prometido. El contrato detalla la forma en la cual sus términos pueden cambiar en algún momento futuro. Sin embargo, como menciona Heymann (2007), una definición precisa de lo que constituye una promesa (o su incumplimiento) involucra problemas no triviales cuando se nota que a través de variables como el "premio por el riesgo", las partes reconocen que los compromisos explícitos no son literalmente incondicionales, pero al mismo tiempo el conjunto de contingencias que llevaría a un default permanece indeterminado. En este sentido surgen dos cuestiones conceptuales relacionadas con las renegociaciones de la deuda.

Primero, si bien en la práctica la mayoría de las deudas son no contingentes, es decir, que su pago no depende del estado de la naturaleza, la posibilidad de renegociar las condiciones del contrato o de hacer default y después llevar a cabo una reestructuración permiten que ex-post el contrato sí dependa del estado de la naturaleza.

Segundo, el hecho de que la deuda soberana pueda ser renegociada hace que la definición de default sea en algún sentido subjetiva. Bajo una definición estricta, si alguno de los pagos prometidos no es llevado a cabo bajo las condiciones originalmente estipuladas el soberano incurriría en un default. Sin embargo, muchas veces las promesas de pagos son renegociadas bajo la amenaza de default, y frecuentemente los acreedores aceptan un pago menor antes que no recibir nada. En este sentido, Grossman y Van Huyck (1988) argumentan que los activos contingentes al estado de la naturaleza pueden ser vistos como defaults parciales "justificados": 
los acreedores observan el estado de la naturaleza y perdonan cierta porción de la deuda condicional a dicho estado. Un punto similar es hecho por Bulow y Rogoff (1988), los cuales argumentan que la deuda soberana puede ser renegociada ex-post bajo la amenaza de sanciones legales. Esto hace que los pagos de la deuda sean condicionales al estado de la naturaleza de facto.

Tomando en cuenta estas consideraciones, es posible hacer una interpretación alternativa de los contratos de deuda soberana. La compra de deuda pública que incluye una prima de riesgo se puede considerar como una apuesta por parte del inversor, ya que el diferencial de tasas de interés muestra que los agentes contemplan concretamente la posibilidad de incumplimientos en algunos estados de la naturaleza. Justamente por la posibilidad de que los soberanos hagan default es que los países emergentes pagan una mayor tasa de interés por sus deudas, de modo de compensar a los acreedores con mayores pagos en los momentos en los que la deuda sí se paga. Bajo esta interpretación, el evento de default -más que una ruptura contractual- es considerado como una de las posibles realizaciones de la "lotería", que por lo general se da en estados de la naturaleza desfavorables para los soberanos.

La interpretación de los contratos de deuda soberana es especialmente relevante en las discusiones sobre reestructuraciones de deuda, en donde a menudo se utilizan argumentos del tipo "ese deudor no es favorable al mercado". La clasificación de un país como "favorable al mercado" o no es ambigua ya que la misma definición se presta a diversas interpretaciones. Una posible definición, basada en el historial de pagos de los países, es que los soberanos que más veces cesaron sus pagos son aquellos menos favorables al mercado. En este contexto, los países con mayor cantidad de defaults podrían considerarse como "deudores recalcitrantes". Este término fue utilizado por Barrington Parker, Juez de la Cámara de Apelaciones de Nueva York, para dar fundamento a un fallo con implicancias económicas. En palabras del Juez: "Es poco probable que ocurran casos como éste en el futuro, porque la Argentina ha sido un «deudor recalcitrante»". De aquí la importancia de definir de manera precisa qué se considera un deudor recalcitrante. Ahora bien, es posible que países que hicieron default le hayan otorgado mayores retornos a sus acreedores que los que hubieran obtenido invirtiendo en su costo de oportunidad, 
ya que durante el tiempo que los soberanos sí cumplieron con sus obligaciones le otorgaron a sus acreedores un premio por el riesgo, y una vez cesados los pagos, posteriormente tuvieron la posibilidad de reestructurar la deuda en default y devolver parte del monto pendiente de pago.

Es posible argumentar que la métrica de interés para los acreedores no es el historial de pagos del soberano per se, sino la rentabilidad que obtuvieron por invertir en dicho activo en comparación con otras inversiones alternativas. Esto motiva una definición alternativa sobre qué se considera como "favorable al mercado". Usando como variable de interés los retornos de la deuda soberana, un país se puede considerar como favorable al mercado si le brindó un retorno ex-post a los acreedores mayor al que hubieran obtenido invirtiendo en un bono libre de riesgo de características similares. Es interesante notar que, bajo esta definición, un país puede ser favorable al mercado incluso con un alto historial de defaults. Si después de cesar sus pagos el país reestructura su deuda y devuelve buena parte de la deuda en default, en el agregado es posible que los inversores terminen obteniendo un retorno mayor al ofrecido por una inversión libre de riesgo. En otras palabras, el historial de default de un país por sí solo no informa si un país está pagando "mucho" o "poco" por su deuda. Entonces, para determinar qué países son "favorables al mercado" o "deudores recalcitrantes" con cierto grado de objetividad, es necesario computar la diferencia entre el retorno de su deuda y el retorno del costo de oportunidad, también conocido como el spread de retornos.

Con el conjunto de spreads soberanos es posible estimar una función de distribución útil para responder diversas preguntas asociadas al debate actual sobre la deuda de los países emergentes, tales como: ¿Cuál ha sido la magnitud promedio de los spreads de retornos ex-post de la deuda soberana de países emergentes en periodos largos de tiempo? ¿Qué proporción de países tuvieron spreads positivos y qué proporción tuvieron spreads negativos? ¿Cómo ha sido la evolución de los spreads a lo largo del tiempo? ¿Cuánto mayor o menor fueron los spreads de los países que hicieron default en comparación con aquellos que no cesaron sus pagos? ¿Cuáles son los países que han brindado spreads atípicamente bajos y atípicamente altos? ¿Hicieron un buen trabajo los mercados de deuda soberana en términos de valuar las compensaciones de riesgo? 
Las respuestas a estas preguntas son importantes para entender las características de la deuda de los países emergentes como clase de activo financiero, pero también pueden tener implicancias acerca del diseño de mecanismos que faciliten las reestructuraciones de deuda pública y las formas de lidiar con los problemas de sobreendeudamiento soberano. El principal aporte de este trabajo consiste en brindar evidencia empírica sobre la distribución de los spreads ex-post que contribuyan a responder las preguntas planteadas en los párrafos anteriores.

Además de esta introducción, el resto del trabajo se estructura de la siguiente manera. En la segunda sección se enmarca el trabajo en la literatura existente y se lleva a cabo una revisión de la literatura empírica que cuantifica diversas dimensiones de la rentabilidad de la deuda soberana. Del análisis de la literatura se desprende que los "recortes" asociados a los episodios de default y reestructuración de deuda han sido, en promedio, de entre el 30\% y $50 \%$ del valor presente de la deuda pendiente de pago. A pesar de esto, a menudo se observan spreads de retornos positivos con respecto a la deuda libre de riego cuando se analizan largos periodos de tiempo -aunque los resultados varían mucho de acuerdo al periodo, país e instrumento de deuda bajo análisis-. Esto se debe a que los retornos altos en los "tiempos buenos" más que compensan las pérdidas asociadas a defaults y sucesivas reestructuraciones.

En la tercera sección se motiva el análisis a partir de la decisión de un agente neutral al riesgo que elige entre invertir entre un bono libre de riesgo y el bono de un soberano riesgoso. De dicha decisión se desprende la ecuación de arbitraje entre el retorno del bono riesgoso y el del bono libre de riesgo, la cual motiva la pregunta de si ambos son iguales ex-post, es decir, si el spread de retornos es igual a cero y en caso de que no lo fuera, si existen diferencias sistemáticas entre periodos, países y características de los países. Para esto se lleva a cabo un análisis empírico con el objeto de construir la función de distribución de los spreads ex-post soberanos de 37 países emergentes en el periodo 1970-2012. En los subsiguientes apartados se documentan las fuentes de información a las que se recurrió y se detalla la metodología de trabajo.

En la cuarta sección se presentan los principales resultados. En primer lugar se muestra que la deuda pública de los países emergentes ha crecido de manera 
importante en las últimos cuatro décadas. Por otro lado, se encuentra que los spreads ex-post de la deuda emergente durante el periodo 1970-2012 fueron, en promedio, 0,29\%. Asimismo, se encuentran los países que brindaron un "exceso de retornos" ex-post atípicamente altos, y que se constituyen como los outliers de la distribución. Estos resultados son robustos a la muestra de países empleada y a diversos supuestos sobre la valuación del stock de deuda en el periodo final. Asimismo, ejercicios de sensibilidad muestran que dichos resultados no varían significativamente ante modificaciones en el precio de mercado de la deuda.

Posteriormente se analiza la evolución en el tiempo de los spreads ex-post y se comparan los retornos de la deuda emergente con los de otro activo riesgoso -el índice Standard \& Poor's 500-. Por una parte se encuentra que los spreads medios han tenido una trayectoria creciente en el tiempo, pasando de ser negativos durante las décadas previas a los 2000 a positivos. Con respecto al spread con la inversión riesgosa se encuentra que, en promedio, la deuda emergente otorgó considerablemente menores, por lo que los spreads ex-post resultaron negativos. Finalmente, se correlacionan los spreads ex-post con medidas de percepciones de los mercados, así como medidas que la literatura usualmente considera como determinantes de los retornos. En ninguno de los dos casos se encuentran asociaciones fuertes, y el poder explicativo de este tipo de variables resultó bajo.

Finalmente, en la quinta sección se ofrecen las reflexiones finales. 


\section{Literatura relacionada}

Este trabajo se relaciona con la literatura que cuantifica empíricamente la rentabilidad de la deuda soberana emitida por países emergentes desde la perspectiva de los acreedores privados. Esta temática ha sido abordada desde dos ángulos distintos.

Por una parte, algunos trabajos han estimado la magnitud de las pérdidas que defaults y reestructuraciones infligen sobre los acreedores. Autores como Jorgensen y Sachs (1989), Suter (1992) y Rieffel (2003) miden dichas pérdidas en alguna dimensión en particular, como la reducción en el valor nominal del stock de deuda, la reducción en el pago de intereses o el alargamiento de los plazos de vencimiento. Sin embargo, para cuantificar correctamente el total de pérdidas que sufren los acreedores como resultado de una reestructuración, se debe comparar el flujo de pagos originalmente prometido con el flujo de pagos de los instrumentos reestructurados, ambos descontados a una tasa de interés común (cf. Sturzenegger y Zettelmeyer, 2007). Sturzenegger y Zettelmeyer (2008) realizan dicho ejercicio para las seis principales reestructuraciones del periodo 1998-2005 y encuentran que el valor presente de los "recortes" estuvo en el rango de entre el $5 \%$ y el $20 \%$ (Uruguay) hasta valores por encima del 70\% (Argentina), mientras que en los restantes países las quitas fueron de entre el $20 \%$ y el $50 \%$.

De manera más reciente, Benjamin y Wright (2013) estiman las quitas asociadas a 112 reestructuraciones de deuda soberana en el periodo 1970-2006, mientras que Cruces y Trebesch (2013) estiman el valor presente de los "recortes" de las 180 reestructuraciones llevadas a cabo por 68 países en el periodo 1970-2010. Estos últimos autores encuentran una gran variabilidad en el tamaño de los "recortes" de acuerdo al país y periodo bajo análisis: la mitad de los "recortes" está por debajo del $23 \%$ o por encima del $53 \%$. El promedio simple de los "recortes" es del $37 \%$, mientras que si se pondera por el volumen total de deuda dicha cifra cae al $30 \%$. Esto significa que, en promedio, los acreedores pudieron preservar casi dos tercios del valor de sus activos en las reestructuraciones.

La principal limitación de estos trabajos es que no brindan información acerca del resultado de los acreedores en periodos largos, es decir, si los defaults y las posteriores reestructuraciones fueron compensados en última instancia por los altos retornos en los periodos "buenos". Para analizar esto, es necesario computar 
el retorno ex-post de los acreedores privados que invirtieron en deuda soberana en periodos largos de tiempo, y comparar dichos retornos con el de su costo de oportunidad, ejercicio que se realiza en este trabajo.

La segunda rama de la literatura relacionada es aquella que computa los retornos ex-post de la deuda soberana y la compara con los retornos de inversiones alternativas. Entre estos trabajos se encuentra el de Eichengreen y Portes (1986), quienes rastrean el historial de pagos de una muestra de bonos soberanos de países emergentes en el periodo 1923-1930, y encuentran spreads ex-post positivos con respecto a los bonos británicos y negativos con respecto a los bonos americanos municipales. Por su parte, Lindert y Morton (1989) computan las tasas internas de retorno de 1.552 bonos emitidos por diez países soberanos en el periodo 1850-1983 y encuentran que los países que brindaron mayores spreads de retornos ex-post a lo largo del periodo fueron Egipto y Argentina, mientras que los países con los menores spreads fueron México y Rusia. Sánchez (1991) realiza este mismo ejercicio, pero aplicado a la deuda pública de Colombia, Ecuador, Perú y Venezuela en el periodo 1840-1914. El autor encuentra que durante el periodo 1840-1870 la región andina en su conjunto tuvo spreads de retornos negativos, lo cual se lo atribuye tanto a los constantes episodios de default como a los altos retornos de los bonos libres de riesgo, sin embargo, durante el periodo 1880-1914 el autor encuentra spreads de retornos ex-post positivos.

De manera reciente, Klingen, Weder y Zettelmeyer (2004) estiman los retornos de la deuda pública de 26 países emergentes en el periodo 1970-2000 y encuentran que el spread ex-post a lo largo del periodo fue negativo el promedio de todos los países en su conjunto. Dicho spread es el reflejo del efecto combinado de la crisis de la deuda de los ochenta (periodo en el cual los retornos de los países emergentes fueron negativos) y retornos altos durante la década de los noventa.

A diferencia de estos trabajos, en donde el foco está puesto en analizar algunos momentos de la distribución de los retornos o de los spreads -como su valor medioen este trabajo se estudia toda la distribución de los spreads, con el fin de investigar cuál fue la proporción de países que tuvieron spreads de retornos positivos y negativos, quiénes fueron los países que brindaron los mayores y menores spreads, cómo fue la evolución en el tiempo de dichos spreads, entre otras cuestiones. 


\section{Estimación de los spreads}

Cuando se considera la decisión de un agente neutral al riesgo que elige entre invertir en un bono libre de riesgo y el bono de un soberano riesgoso, se obtiene la ecuación de arbitraje entre los retornos de ambos bonos ${ }^{1}$ :

$$
\underbrace{1+i^{*}}_{\begin{array}{c}
\text { Retorno esperado de un } \\
\text { bono libre de riesgo }
\end{array}}=\underbrace{(1-p)(1+i)+p \phi}_{\begin{array}{c}
\text { Retorno esperado de un } \\
\text { bono riesgoso }
\end{array}}
$$

En donde $i^{*}$ es la tasa de interés del bono libre de riesgo, $i$ es la tasa de interés del bono del soberano riesgoso, $p$ es la probabilidad de que el soberano haga default y $\phi$ es la tasa de recupero esperada, condicional a que el soberano haya hecho default.

Esta condición establece que ex-ante los retornos esperados de un bono riesgoso y uno libre de riesgo deben ser iguales, de lo contrario existirían oportunidades de obtener ganancias a través de operaciones de arbitraje. Sin embargo, no brinda información acerca de si retornos realizados o ex-post son iguales o no. En principio, existen varias razones por las cuales la ecuación (1) puede no ser igual ex-post, tales como impuestos a las transacciones financieras, mercados internacionales de crédito no competitivos, costos de transacción, restricciones al movimiento de capitales, entre otras. En este sentido, divergencias en la ecuación de arbitraje señalizarían fricciones en los mercados.

Por otro lado, desviaciones en la ecuación de arbitraje pueden también ser consecuencia de errores sistemáticos de pronóstico. Esto se puede reflejar en que los acreedores perciban distribuciones de probabilidad de default distintas de las verdaderas o en que el valor de recupero que esperado después de un default sea mayor al que efectivamente recibido. La lógica detrás de estas desviaciones sistemáticas es que la capacidad de repago de los soberanos es incierta, especialmente en los países emergentes, los cuales son más volátiles. Hay estados de la naturaleza en los cuales los países no pueden honrar sus deudas, y es justamente por eso que los países pagan una tasa de interés sobre su deuda que incluye una compensación sobre

\footnotetext{
${ }^{1}$ Esta condición surge del proceso de maximización de beneficios de los inversores, como se demuestra en el Apéndice A. La notación de la ecuación de arbitraje se encuentra simplificada para facilitar la lectura.
} 
el riesgo. Dada la dificultad de estimar los riesgos, estos a menudo son calculados erróneamente. Como menciona Heymann (2007), la estimación de tasas de retorno de largo plazo y de capacidades de pago descansa sobre modelos de comportamiento económico inciertos. Las variables fundamentales que determinan el desarrollo de las economías en procesos donde las estructuras y las instituciones sufren cambios posiblemente irreversibles no están claramente identificadas, medidas y proyectadas. En otras palabras, predecir correctamente el valor de las variables en el futuro en base a la historia reciente conlleva una gran dificultad. ${ }^{2}$ Reinhart y Rogoff (2009, p. 287) proveen un ejemplo ilustrativo cuando en el 2009 clasificaron a Grecia como un país candidato a "graduarse", es decir, un país que "logró salir de siglos de defaults constantes sobre su deuda soberana y eventualmente dejó de hacer default" (p. 283).

En caso de que ex-post la ecuación de arbitraje no se cumpla con igualdad, la diferencia entre el lado izquierdo y el lado derecho de la ecuación constituye la siguiente función:

$$
s_{i} \equiv r_{i}-r_{i}^{*}
$$

En donde $s_{i}$ es el spread o diferencial de retornos, $r_{i}$ es la tasa de retorno ex-post de la deuda del soberano $i$, y $r_{i}^{*}$ la tasa de retorno que hubieran obtenido los acreedores si en vez de haber invertido en el soberano $i$ lo hubieran hecho en deuda libre de riesgo. ${ }^{3}$ Es importante notar la diferencia entre las ecuaciones (1) y (2). Mientras que la ecuación (1) se refiere a los retornos esperados, la ecuación (2) se refiere a los retornos realizados. En otras palabras, ex-ante se espera que se cumpla

\footnotetext{
${ }^{2}$ Dado que el precio del bono es una función del flujos de fondos futuro, así como de la probabilidad esperada de que el soberano haga default y del valor de recupero en caso de que este reestructure su deuda, los inversores deberían proyectar cada variable, en cada momento del tiempo, para cada estado de la naturaleza.

${ }^{3}$ Considerando que en los hechos cada soberano recibió una serie de préstamos diferente, y que la rentabilidad de la deuda libre de riesgo también varió en el tiempo, el costo de oportunidad de los acreedores no es único, sino que varía de acuerdo a cada país. Para que los costos de oportunidad de dos deudores soberanos sean iguales, las secuencias de financiamiento que recibieron ambos países tendrían ser las mismas o la tasa libre de riesgo debería de mantenerse constante en el tiempo. En los datos ninguna de las dos cosas ocurrió.
} 
la ecuación de arbitraje con igualdad, mientras que en este trabajo se estima lo que sucedió ex-post, una vez que ocurrieron las secuencias de pagos. En términos prácticos, para construir la distribución de los spreads, primero es necesario estimar los retornos ex-post de la deuda pública de cada país, y posteriormente compararlos con los retornos de la deuda libre de riesgo. Una vez estimados los retornos de la deuda soberana y los retornos de la deuda libre de riesgo, el spread ex-post de cada soberano queda determinado como la diferencia entre ambos retornos.

Al resolver la ecuación (2) para cada soberano, es posible estimar la función de distribución del conjunto de spreads. A partir del análisis de dicha distribución se puede determinar si los mercados financieros hacen -en promedio- un buen trabajo en valuar las compensaciones por el riesgo; si la valuación de los riesgos difiere sistemáticamente de acuerdo al periodo bajo análisis o de acuerdo a las características de cada país, como su nivel de ingreso o su historial de pagos. También permite analizar cómo ha sido la evolución de la distribución de los spreads a lo largo del tiempo, analizar qué proporción de países tuvieron spreads de retornos positivos y negativos, y determinar qué países son los que brindaron spreads de retornos atípicamente altos y atípicamente bajos. Las fuentes de información y la metodología para estimar empíricamente la función de distribución del spread de retornos ex-post se aborda en las siguientes subsecciones.

\subsection{Fuentes de información}

En esta subsección se detallan las fuentes de información que se utilizan a lo largo del trabajo para estimar la función de distribución del spread de retornos ex-post de la deuda pública en manos de acreedores privados de una muestra de países emergentes.

La información acerca del endeudamiento soberano se obtuvo de la base de datos Estadísticas de la Deuda Internacional (IDS, por sus siglas en inglés) del Banco Mundial. ${ }^{4}$ De dicha base se obtuvo información de 37 países emergentes en el periodo

\footnotetext{
${ }^{4} \mathrm{La}$ IDS provee información sobre el stock deuda soberana y los flujos financieros de todos los países que reportan al "Sistema de Notificación de la Deuda" del Banco Mundial. Este sistema funciona desde 1951 y registra información a nivel de préstamos individuales, aunque solamente publica los datos a nivel agregado. Todos los países que reciben préstamos por parte del Banco
} 
1970 - 2012 sobre su stock de deuda soberana, pago de intereses, amortizaciones de capital, desembolsos, flujos netos, transferencias netas, vencimientos promedio de las nuevas emisiones de deuda, intereses atrasados, capitalización de intereses, condonaciones de la deuda y composición por monedas del stock de deuda. En el Apéndice B se ofrecen las definiciones de cada uno de estos conceptos. Se obtuvieron datos de cada una de estas variables para el total de la deuda soberana en manos de acreedores privados y sus tres componentes: bonos, préstamos de bancos comerciales y financiación de otros acreedores privados. Los datos están en dólares corrientes de Estados Unidos. El Ingreso Nacional Bruto (INB) se obtuvo de la base de datos Indicadores del Desarrollo Mundial del Banco Mundial; el tipo de cambio nominal de la base Estadísticas Financieras Internacionales del FMI; la tasa de rendimiento de las letras del tesoro norteamericano a diez años de la Reserva Federal (FED) de Estados Unidos. Finalmente, los precios en el mercado secundario de los instrumentos de deuda se obtuvieron de Klingen, Weder y Zettelmeyer (2004) para el periodo 1970-2001 y de Bloomberg para los años posteriores.

Es importante aclarar que la base de datos IDS sólo provee información para deuda de largo plazo (aquella con un vencimiento superior a un año al momento de emisión), por lo tanto, todos los resultados de este trabajo corresponden a deuda de largo plazo. En la práctica casi toda la deuda soberana es emitida con una madurez superior a un año. Asimismo, el concepto utilizado de deuda soberana incluye tanto a la deuda del sector público como a la deuda del sector privado que tiene una garantía por parte del sector público. Sólo se considera a la deuda soberana en manos de acreedores privados, y no en manos de organismos multilaterales u otros países.

En el Apéndice C se muestra el listado de países que incluye la muestra, así como estadísticas descriptivas sobre los defaults y las reestructuraciones que llevaron a cabo durante el periodo 1970-2012. Como se puede apreciar, la muestra es muy diversa. El $44 \%$ de los países de la muestra (18 en total) nunca cesó sus pagos en el periodo bajo análisis. El $41 \%$ de los países hizo un default sobre su deuda, el $12 \%$ dos defaults y un país (Ecuador) hizo tres defaults. De los países que hicieron

Mundial acuerdan proveer información acerca de su deuda externa a este sistema. 
defaults y posteriormente reestructuraron su deuda, el recorte promedio - medido como disminución en el valor presente del stock nominal de la deuda- es del $30 \%$.

\subsection{Metodología}

El desafío consiste en estimar la rentabilidad de la deuda soberana de cada país y su costo de oportunidad asociado. Para estimar la rentabilidad de la deuda, la literatura empírica ha empleado básicamente dos metodologías distintas.

El primer método se basa en rastrear el historial de pagos de cada emisión de deuda individual emitida por cada país, computar la tasa interna de retorno del flujo de pagos de dicho instrumento y promediar los retornos resultantes. Este es el enfoque que utilizan Eichengreen y Portes (1986) y Lindert y Morton (1989). Dicha metodología presenta algunos inconvenientes. En primer lugar, es sumamente difícil rastrear el historial de pagos de todos los instrumentos de deuda emitidos por cada país en cada año, especialmente aquellos emitidos hace varias décadas. Asimismo, la disponibilidad de esta clase de información para los países chicos es limitada, ya que muchos de ellos no tienen un instituto de estadísticas organizado y confiable. Por último, no hay una base de datos pública que ofrezca información a nivel individual de cada emisión de deuda por parte de los soberanos, por lo cual los trabajos que utilizan esta metodología son difícilmente replicables.

La segunda forma de estimar los retornos ex-post de la deuda pública consiste en calcular las tasas internas de retornos en base a los flujos de pagos agregados entre los acreedores privados y los deudores soberanos, aplicando la misma metodología que en la evaluación de un proyecto de inversión. Este es el enfoque que sigue Lindert (1989) y Kligen, Weder y Zettelmeyer (2004), y el cual se emplea en este trabajo. Una ventaja de esta metodología es que la información necesaria para llevarla a cabo es publicada por el Banco Mundial. Sin embargo, también presenta algunos inconvenientes.

En primer lugar, como observan Klingen et al. (2004), para poder calcular las tasas internas de retornos de la deuda soberana a partir de los flujos financieros publicados por el Banco Mundial es necesario realizar algunos ajuste sobre la serie de transferencias netas. El problema subyacente es que las reestructuraciones de deuda que involucraron intercambios entre distintas clases de activos sesgan las 
tasas de retorno. Para solucionar este problema es necesario construir una serie de transferencias netas modificadas que tome en cuenta los intercambios entre clases de activos. En el Apéndice D se explica de manera más pormenorizada el problema, así como los pasos llevados a cabo para corregirlo.

El segundo problema de este enfoque es que la analogía con la evaluación de un proyecto de inversión no es del todo precisa. Por una parte, no hay algo equivalente a un pago final en el último periodo de la muestra sino que, al final de la muestra, los acreedores tienen en su posesión un stock de deuda pendiente de cobro, es decir, un derecho a (potencialmente) recibir futuras amortizaciones de capital y pago de intereses. De manera similar, en el primer año de la muestra los acreedores no realizan una inversión inicial, sino que al inicio de la muestra ya poseen un stock de deuda por cobrar. Este último punto no es un gran problema para computar las tasas de retorno ya que el stock de deuda inicial en la muestra es muy pequeño en comparación con los desembolsos de los años posteriores, pero sí es un problema para computar las tasas de retornos en subperiodos.

Para solucionar este segundo inconveniente, se sigue la solución propuesta por Klingen et al. (2004), los cuales valúan tanto el stock de deuda inicial como el stock de deuda final a los precios del mercado secundario. En otras palabras, se computan las tasas de retorno como si los inversores hubieran comprado el stock de deuda inicial en el mercado durante el primer periodo, y después de recibir una serie de "transferencias netas" vendieran su stock de deuda final a los precios vigentes en el mercado. Es importante notar que los precios en el mercado secundario están disponibles a partir de mediados de los ochenta y sólo para algunos instrumentos de deuda. Adicionalmente, en la medida en la que se elija la muestra de países en base a la disponibilidad de precios en el mercado secundario algunos resultados se exponen a un sesgo de selección ya que es posible que los países que tuvieron peor desempeño desaparecieron de los mercados de deuda soberana. Nuevamente, en el Apéndice D se brinda mayor detalle acerca de la valuación a precios de mercado del stock de deuda soberana.

El concepto de "transferencias netas" que provee la base IDS posee un rol central en el cálculo de las tasas internas de retorno, ya que captura tanto los nuevos fondos que recibe el soberano como los servicios de la deuda que debe pagar. 
Empleando este concepto, los defaults y las operaciones de reestructuración se reflejan de la manera esperada sobre las tasas de retorno. A manera de ejemplo, un default en los pagos de la deuda y su posterior reestructuración tienen como consecuencia que las amortizaciones de capital se paguen en periodos posteriores a los acordados. Dado que el valor presente de dicha amortización es menor, una reestructuración se refleja en menores tasas internas de retornos.

En términos analíticos, la tasa de retorno ex-post de la deuda pública del soberano $i, r_{i}$, en el periodo comprendido entre $t_{0}$ y $T$ es la que resuelve la siguiente ecuación:

$$
-D_{i t_{0}}+\sum_{t=t_{0}}^{T} \frac{T N_{i t}}{\left(1+r_{i}\right)^{t}}+D_{i T}=0
$$

en donde $D_{i t_{0}}$ representa el valor de mercado del stock de deuda en el año inicial del periodo bajo análisis, $T N_{i t}$ las transferencias netas que recibe el acreedor en el periodo $t, D_{T}$ el valor de mercado del stock de deuda en el último año de la muestra y el subíndice $i$ indexa a los distintos países. Para estimar la tasa interna de retorno ex-post se resuelve la ecuación (3) para el total de deuda soberana en manos de acreedores privados cada soberano $i$, tomando como $t_{0}=1970 \mathrm{y}$ $T=2012$. Asimismo, esta ecuación también se resuelve para cada instrumento de deuda (bonos, préstamos bancarios y otros acreedores privados), y para distintos subperiodos.

Una vez estimado el retorno de la deuda soberana, para calcular el spread es necesario comparar dicho retorno con su costo de oportunidad, suponiendo que los fondos netos que fueron hacia los mercados de deuda emergentes hubieran ido a parar a activos alternativos. La comparación que se realiza es con el retorno de un instrumento de deuda de similar vencimiento, pero presuntamente libre de riesgo de default. En la base de datos empleada, el vencimiento promedio de la deuda soberana es de 10,1 años. Por lo tanto, como activo alternativo libre de riesgo se utiliza a las letras del tesoro de Estados Unidos a diez años. ${ }^{5}$

\footnotetext{
${ }^{5}$ Como notan Klingen et al. (2004), el uso del vencimiento promedio de la deuda no está exento de problemas. La razón es que una fracción importante de dicha deuda -en promedio, un
} 
Como se mencionó previamente, computar el retorno promedio anual de la deuda libre de riesgo sería un error ya que dicho cálculo no toma en cuenta el patrón temporal específico de entradas y salidas de capital que tuvieron los países emergentes. En los hechos, la mayoría de los flujos hacia los países emergentes ocurrieron durante la década de los 2000, cuando la inflación de Estados Unidos y el retorno de sus bonos públicos fueron relativamente bajos, por lo cual, al momento de computar el retorno del costo de oportunidad debería dársele una mayor ponderación a este periodo. El desafío consiste en construir un flujo de fondos alternativo, en el cual se simula que los desembolsos efectivamente observados hubieran sido invertidos en letras del tesoro, y estimar la rentabilidad sobre dicha simulación.

Para brindar mayor claridad sobre este punto, supóngase que el activo alternativo es un bono libre de riesgo cupón cero del tesoro norteamericano, de vencimiento $\tau \geq 1$. Sea $t_{0}$ el año en el cual el primer bono fue comprado y $T$ el año en el cual el último bono fue comprado. Supóngase también que en los siguientes años también hay compras de letras del tesoro y que dichos activos son mantenidos hasta su vencimiento. Entonces, las transferencias netas de la inversión alternativa desde la perspectiva del acreedor se definen como:

$$
T N_{t}^{A}=d_{t}^{A}-\left(1+i_{t-\tau}\right)^{\tau} d_{t-\tau}^{A}, \quad t \in\left[t_{0}, T+\tau\right]
$$

en donde $d_{t}^{A}$ son los desembolsos a la inversión alternativa $A$ en el periodo $t, \mathrm{y}$ $i_{t}$ es el rendimiento al vencimiento de la inversión en el periodo $t$. Como ningún bono fue comprado antes del periodo $t_{0}, d_{t}^{A}=0$ para $t<t_{0}$ y $T N_{t_{0}}^{A}=d_{t_{0}}^{A}$. Adicionalmente, dado que no se compran bonos después del periodo $T, d_{t}^{A}=0$ para $t>T$. El punto está en construir los flujos del lado derecho de la ecuación anterior usando información sobre transferencias netas y stocks de deuda. En la

tercio del total en la muestra empleada- incluye una tasa de interés flotante (típicamente definida como un margen sobre la tasa LIBOR a seis meses). Para este tipo de deuda el deudor es el que asume el riesgo de que haya cambios en la tasa de interés internacional, mientras que el valor de la deuda no varía en gran medida ante cambios en la tasa de interés. Por lo tanto, la sensibilidad del precio de la deuda ante desplazamientos en la curva de retornos, lo que se conoce como la "duración efectiva", es cercana a cero. La "duración efectiva" promedio debe estar entre seis meses y diez años. Desafortunadamente, no hay datos disponibles sobre la duración de los préstamos privados a deudores soberanos a lo largo del periodo. 
muestra, el primer desembolso que reciben los soberanos corresponde al stock de deuda inicial. En la simulación se presume que dicho desembolso fue a parar al activo alternativo. Posteriormente, se simula que los "flujos netos" de las letras del tesoro son los mismos que los "flujos netos" hacia los mercados emergentes. Dado que las amortizaciones en el periodo $t, a_{t}$, son equivalentes a los desembolsos $t-\tau$ periodos atrás $\left(a_{t}=d_{t-\tau}^{A}\right)$ se tiene que:

$$
T N_{t}^{A}=d_{t}^{A}-\left(1+i_{t-\tau}\right)^{\tau} d_{t-\tau}^{A}, \quad t \in\left[t_{0}, T+\tau\right]
$$

Lo cual permite definir las series de manera recursiva, usando las siguientes igualdades:

$$
d_{t}^{A}-d_{t-\tau}^{A}=d_{t}-a_{t}=D_{t}-D_{t-1}, \quad t \in\left(t_{0}, T\right]
$$

en donde $D_{t}$ representa al stock de deuda soberana en el periodo $t$. Asimismo, es posible computar los retornos anuales de activo alternativo usando un "índice de retornos". En el caso de las letras del tesoro a 10 años el índice de retornos refleja dos componentes: el cupón prometido al inicio del año y el cambio en el precio del bono como consecuencia de cambios en la tasa de interés. Las transferencias netas de los activos alternativos se computan asumiendo que se mantienen por un año. Sea $\rho_{t}$ el retorno anual en el periodo $t$, es decir, la diferencia porcentual entre el índice de retornos al final del periodo $t \mathrm{y}$ al final del periodo $t-1$. Las transferencias netas en el periodo $t$ son:

$$
\begin{aligned}
T N_{t}^{A} & =d_{t}^{A}-\left(1+\rho_{t}\right) d_{t-1}^{A}, \quad \text { para } \quad t \in\left[t_{0}, T\right] \\
d_{0}^{A} & =p_{0} D_{0}, \quad \text { para } \quad t \leq t_{0} \\
d_{t}^{A} & =D_{t}-D_{t-1}+d_{t-\rho}, \quad \text { para } \quad t \in\left(t_{0}, T\right) \\
d_{t}^{A} & =0, \quad \text { para } \quad t \geq T
\end{aligned}
$$

Finalmente, se computan las tasas internas de retornos sobre estos flujos de fondos simulados, aplicando la misma metodología que para la deuda pública. Una vez que obtenidas los retornos de la deuda de cada soberano y su respectivo costo de oportunidad, el spread de retornos se computa simplemente como la diferencia entre ambos. Los resultados se presentan en la siguiente sección. 


\section{Resultados}

En el Gráfico 1 se muestra la evolución del stock de deuda pública en manos de acreedores privados y la evolución de las transferencias netas en las últimas cuatro décadas para el total de la muestra.

Gráfico 1. Stock de deuda soberana y transferencias netas. 1970-2012

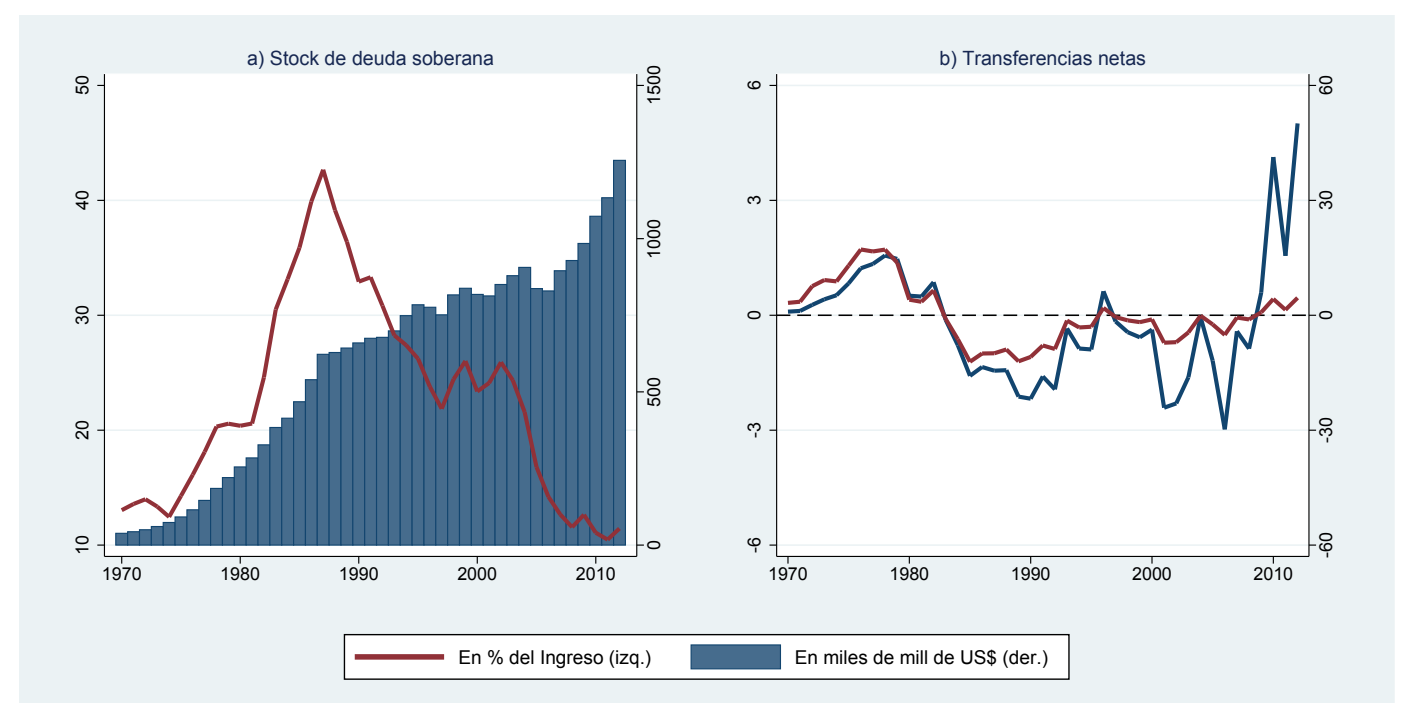

Fuente: elaboración propia en base a datos del Banco Mundial.

Como se puede ver en el panel a), en términos absolutos el stock de deuda externa en manos de acreedores privados ha crecido notablemente en los últimos 40 años, pasando de 38 mil millones de dólares en 1970 a más de 1200 miles de millones en el 2012. Sin embargo, en términos del ingreso mundial, la deuda pública de los países emergentes alcanzó sus máximos valores durante la crisis de la deuda de los ochenta, llegando a representar hasta un $42 \%$ del ingreso mundial durante 1987. A partir de dicho año la deuda soberana ha visto una tendencia decreciente hasta representar el 11\% de los ingresos en la actualidad. Esto se debe a que, con la excepción de algunos episodios puntuales, el crecimiento mundial del ingreso fue notablemente superior al del stock de deuda a lo largo de las últimas dos décadas. En el panel b) se muestra la evolución de las transferencias netas desde la perspectiva de los países. Durante gran parte de la década de 1970 y los 2000 las transferencias netas fueron positivas. Esto significa que los soberanos fueron 
receptores netos de capitales, es decir, que los desembolsos que recibieron fueron superiores a los pagos de deuda. Con la crisis de los ochenta, los países emergentes dejaron de recibir fondos y las transferencias netas tuvieron una fuerte caída. En el agregado, las transferencias netas fueron negativas durante la mayor parte de la década de los ochenta y noventa.

\subsection{Retornos de la deuda y spreads soberanos}

El retorno promedio de la deuda soberana durante el periodo 1970-2012 fue del 9,5\%, mientras que el mediano del 8,9\%. Sin embargo, hay una notable heterogeneidad entre países y regiones. Al analizar los retornos de acuerdo al nivel de ingresos el patrón es claro: mayores ingresos promedio se asocian a mayores retornos ex post, aunque las diferencias son pequeñas. Los países de ingresos medios-altos tuvieron retornos promedio del 10,2\%, los de ingresos medios-bajos del 8,6\% y los de ingresos bajos del 7,6\%. En términos regionales, América Latina junto con Europa emergente y Asia Central fueron las regiones con mayores retornos $(11,2 \%$ y $9,4 \%$, respectivamente), mientras que las regiones con menores retornos fueron el Este y el Sur de Asia (7,8\% y 7,6\%, respectivamente). Con respecto a los retornos de acuerdo al monto endeudado, se encuentra que el diez por ciento de los países más endeudados (en términos de su ingreso) brindó retornos muy similares al diez por ciento con menor deuda (8,4\% y $9,1 \%$, respectivamente). Interesantemente, los retornos ex-post de los países que hicieron default de su deuda una vez (10,6\%) o dos veces $(10,7 \%)$ fueron mayores que los retornos de los países que no hicieron default $(7,9 \%)$ durante el periodo bajo análisis.

Ahora bien, para determinar si estas magnitudes son "altas" o "bajas", los retornos se deben comparar con su costo de oportunidad. Para esto, se calcula la diferencia de los retornos de la deuda soberana con los de las letras del tesoro americano a diez años. En el Gráfico 2 se muestran los spreads ex-post de toda la muestra para el periodo 1970-2012.

El 65 por ciento de los países de la muestra tuvo un spread negativo, mientras que el 35 por ciento tuvo valores por encima del cero. El spread medio (mediano) de toda la muestra fue del $0,3 \%(-0,2 \%)$. El hecho de que dichos spreads sean tan cercanos a cero es evidencia de que los mercados de deuda soberana hicieron un buen 
Gráfico 2. Spreads ex-post con respecto a las letras del tesoro a 10 años. 1970-2012

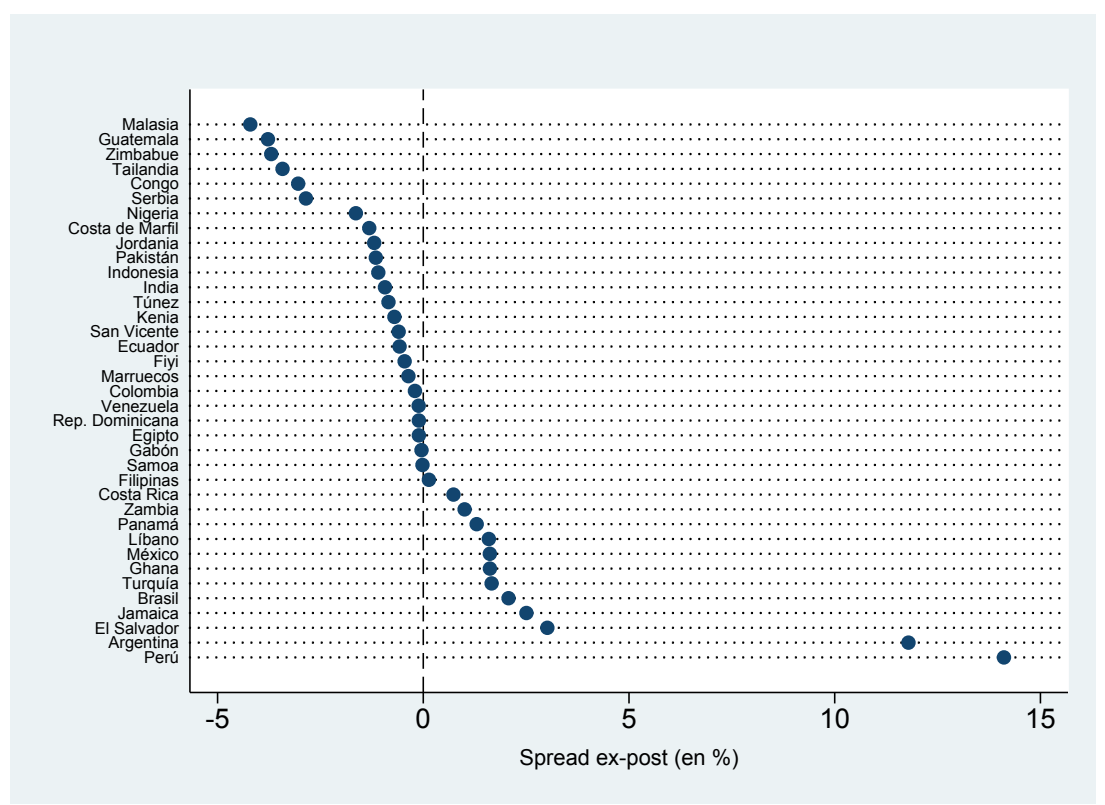

Fuente: elaboración propia en base a datos del Banco Mundial.

trabajo en compensar los riesgos asociados a este tipo de inversión. En la siguiente subsección se muestra que este resultado es robusto en diversas dimensiones.

Al desagregar el análisis por regiones se observa que América Latina fue la que otorgó mayores excesos de retornos en promedio $(2,2 \%)$, mientras que el Este de Asia fue la región con menores spreads (-1,5\%). Al analizar a los países agrupándolos de acuerdo a su nivel de ingreso se observa que los países de ingresos medios-altos fueron los únicos que brindaron excesos de retornos positivos $(1,2 \%)$, los de ingresos medios-bajos tuvieron spreads cercanos a cero $(0,7 \%)$, mientras que el peor desempeño de todos lo tuvo el grupo de países de ingresos bajos, con retornos notablemente por debajo de la inversión alternativa $(-2,2 \%)$.

Con respecto a la relación entre los spreads y las variables de endeudamiento, en primer lugar se encuentra que el diez por ciento más endeudado tuvo un spread positivo $(0,8 \%)$, mientras que el diez por ciento con menos deuda tuvo un spread negativo $(-1,0 \%)$, aunque no hay una relación monotónica entre cantidad endeudada y el spread ex-post. Por su parte, los países que hicieron default dos o más veces tuvieron mayores spreads $(2,0 \%)$ que aquellos que sólo hicieron un default sobre su 
deuda $(1,0 \%)$ o ninguno (-1,0\%). En la siguiente sección se realiza un detalle más pormenorizado de la relación entre las variables de deuda y el spread ex-post.

Un punto final a analizar se refiere a qué países fueron los que brindaron spreads atípicamente altos y atípicamente bajos o, en otras palabras, quiénes fueron los outliers de la distribución de spreads. Es importante notar que no necesariamente los países con mayores retornos son aquellos con mayores spreads ex-post. Si bien la correlación lineal entre los retornos y los spreads es alta $(0,93)$, la correlación de Spearman entre el ranking de los países dista de ser perfecta (0,70). En el Gráfico 3 se presentan estimaciones no paramétricas calculadas por el método de Kernels de la función de densidad del "exceso de retornos" con respecto a la deuda libre de riesgo.

Gráfico 3. Distribución de los spreads ex-post. 1970-2012

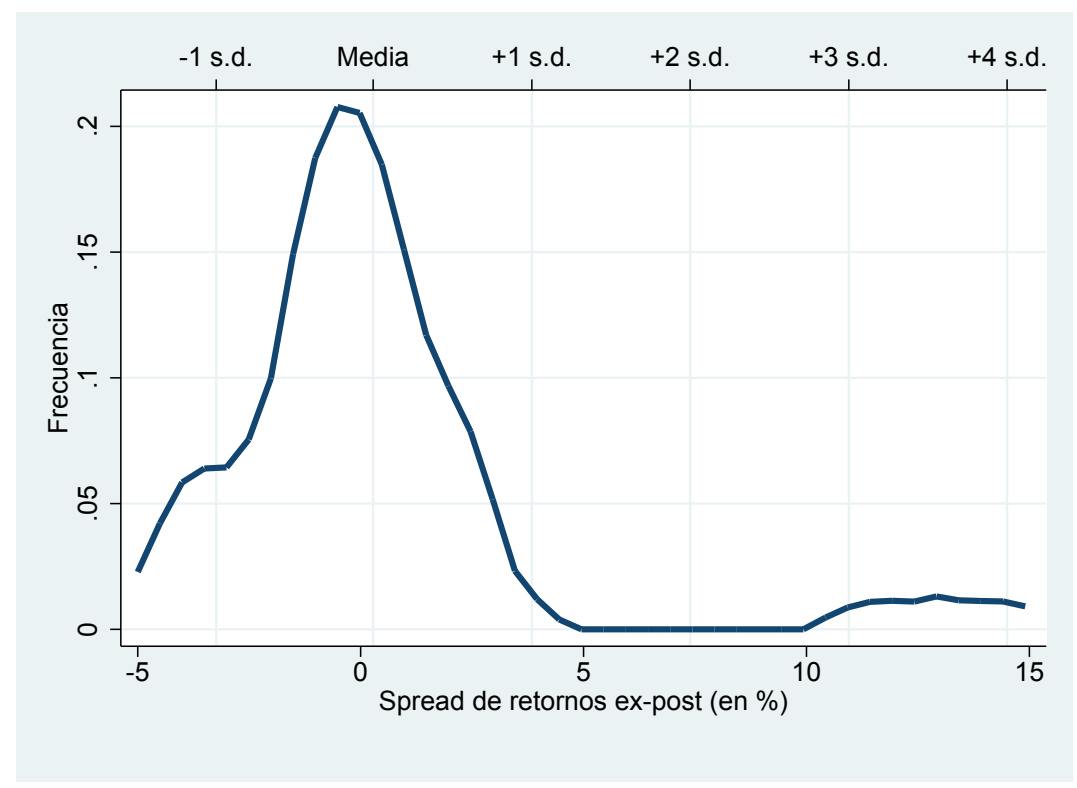

Fuente: elaboración propia en base a datos del Banco Mundial.

Si consideramos como un outlier del default a aquellos países con un "exceso de retornos" sobre el activo libre de riesgo al menos un desvío estándar por encima o por debajo de la media, entonces, estos los outliers son Malasia $(-4,2 \%)$ del lado negativo de la distribución y Argentina (11,7\%) y Perú (13,2\%) del lado positivo. De hecho, estas observaciones son tan atípicas que conducen parte de los resultados agregados: si excluimos a los outliers de los resultados, el spread de la media 
muestral pasa a ser negativo (-0,3\%), y el spread de América Latina cae casi dos puntos porcentuales, de $2,2 \%$ a $0,4 \%$, aunque sigue siendo la región con mayores spreads. En la próxima subsección se analiza cuán robustos son estos resultados ante un posible sesgo de selección de los países y antes diferentes supuestos sobre los precios de la deuda. Asimismo se realiza un análisis de sensibilidad de los spreads antes modificaciones en el precio de la deuda.

\subsection{Robustez de los resultados y análisis de sensibilidad}

En primer lugar se analiza la robustez de los resultados ante un posible sesgo de selección en la muestra. La base del IDS tiene disponible datos desde 1970 para 83 países, de los cuales hay información sobre precios de mercado tan sólo para 37. Considerando que este subconjunto de países define la muestra, los resultados agregados podrían mostrar un sesgo de selección ya que los países que tuvieron peor desempeño en términos de retornos -desde el punto de vista de los acreedores-, posiblemente sean aquellos cuyos bonos dejaron de negociados en los mercados de deuda secundarios.

Para lidiar con este problema seguimos el enfoque de Klingen et al. (2004). Se muestran los resultados para los países que tienen precios disponibles en 1991, suponiendo que el precio de la deuda de dichos países es cero en el 2012. La lógica de este procedimiento es la siguiente: antes de 1992 la presencia de un mercado secundario de deuda no era un signo de "solidez" de la deuda soberana. El mercado secundario de la deuda pública fue creado durante la década de 1980 para comerciar préstamos de países que tenían grandes stocks de deuda y que habían tenido dificultades para pagar los servicios de su deuda durante los 80's. En 1991 ningún país había salido del mercado secundario (el primero en hacerlo fue Bolivia en 1993). Por lo tanto, permitir que la existencia de precios en el mercado secundario en 1991 defina nuestra muestra no crea un sesgo de los retornos. Dado que asumimos que el precio en el 2012 es cero, las tasas de retorno computadas bajo este supuesto representan una cota inferior de la verdadera tasa de retorno de dicha muestra.

En segundo lugar, se estudia la robustez de los resultados ante distintos supuestos sobre los precios de la deuda. Por una parte, se analiza cómo se modifican los 
resultados si en vez de usar los precios del 2012 para valuar el stock final de deuda se utilizan los precios del 2011. Por otro lado se realiza un ejercicio de imputación de precios. Como se explica en detalle en el Apéndice D.2, los precios de la deuda soberana se calculan en base a una muestra de bonos para los cuales hay precios disponibles en el mercado secundario de deuda. El ejercicio de robustez que se realiza consiste en imputarle precios a los bonos soberanos que no tuvieron precio en el 2012. Esta imputación fue hecha en base al método de "estratificación"6. Una vez construidos los estratos e imputados los precios de los bonos, se calculó nuevamente el valor del stock de deuda con los nuevos precios y estimó el spread de deuda soberano.

Finalmente, se analiza la sensibilidad de los resultados ante variaciones en el precio de la deuda en el último periodo. En particular, se estudia cómo se modifican los spreads si el stock de deuda fuera valuado a la par (es decir, como si el mercado no hiciera ningún descuento) y cómo cambian los spreads ante disminuciones e incrementos del $15 \%$ y $25 \%$ por ciento en el valor del precio final. Los resultados de los ejercicios de robustez y sensibilidad se resumen en el Cuadro 2.

En la columna (1) del Cuadro 2 se muestran los resultados considerando el posible sesgo de selección. En este caso la muestra incorpora tres países adicionales que no tienen precios de mercado en el 2012 pero sí en 1991: Argelia, Bolivia y la República Democrática del Congo. El principal resultado es que el sesgo de selección no tiene un gran efecto sobre la media de los spreads de retornos: el promedio se modifica menos de un punto porcentual, de 0,29\% a 0,89\%. En este caso, el 40 por ciento de los países tienen spreads positivos. En las columnas (2) y (3) se muestran los resultados ante distintos supuestos de los precios de la deuda: utilizando los precios del 2011 para valuar el stock final de deuda e imputando precios para los bonos que no tienen precios en el mercado. En ambos casos se observa que los spreads se modifican sólo marginalmente, pasando a 0,30\% cuando se usan los precios del 2011 y a 0,24\% cuando se realiza una imputación de precios. De igual manera, el ranking de países en términos de la magnitud de sus

\footnotetext{
${ }^{6}$ Este método consiste en formar "estratos" o grupos de bonos que sean homogéneos de acuerdo a algunas variables de interés, y posteriormente imputarle a los bonos sin precio el precio promedio de los bonos de características similares. Los estratos fueron construidos a nivel país de acuerdo a la monedad denominación del bono, el periodo vencimiento y la amortización pendiente de pago.
} 
Cuadro 2. Robustez de los resultados ante distintos escenarios. (en \%)

\begin{tabular}{|c|c|c|c|c|c|c|c|c|}
\hline & $\begin{array}{l}\text { Sesgo sel. } \\
\text { (1) }\end{array}$ & $\begin{array}{c}\text { Precio } 2011 \\
\text { (2) }\end{array}$ & $\begin{array}{c}\text { Imputación } \\
(3)\end{array}$ & $\begin{array}{c}\text { A la par } \\
(4)\end{array}$ & $\begin{array}{c}\text { Menos } 25 \% \\
\text { (5) }\end{array}$ & $\begin{array}{c}\text { Menos } 15 \% \\
(6)\end{array}$ & $\begin{array}{c}\text { Más } 15 \% \\
(7)\end{array}$ & $\begin{array}{c}\text { Más } 25 \% \\
(8)\end{array}$ \\
\hline Todos & 0,89 & 0,30 & 0,24 & 0,14 & $-0,20$ & 0,01 & 0,53 & 0,70 \\
\hline África al Sur del Sahara & 0,68 & $-0,92$ & $-0,98$ & $-1,04$ & $-1,16$ & $-1,08$ & $-0,88$ & $-0,81$ \\
\hline Congo & $-3,04$ & $-3,15$ & $-3,04$ & $-2,95$ & $-3,18$ & $-3,12$ & $-2,96$ & $-2,91$ \\
\hline Costa de Marfil & $-1,31$ & $-1,40$ & $-1,35$ & $-1,29$ & $-1,37$ & $-1,34$ & $-1,28$ & $-1,26$ \\
\hline Gabón & $-0,04$ & $-0,10$ & $-0,04$ & $-0,25$ & $-0,35$ & $-0,22$ & 0,12 & 0,22 \\
\hline Ghana & 1,62 & 1,51 & 1,62 & 1,31 & 1,01 & 1,28 & 1,92 & 2,10 \\
\hline Kenia & $-0,70$ & $-0,76$ & $-0,70$ & $-0,67$ & $-0,86$ & $-0,80$ & $-0,61$ & $-0,55$ \\
\hline Nigeria & $-1,64$ & $-1,64$ & $-1,64$ & $-1,64$ & $-1,65$ & $-1,64$ & $-1,64$ & $-1,63$ \\
\hline Rep. Dem. Congo & 13,97 & n.d. & n.d. & n.d. & n.d. & n.d. & n.d. & n.d. \\
\hline Zambia & 1,00 & n.d. & 1,00 & 1,00 & 0,92 & 0,95 & 1,06 & 1,09 \\
\hline Zimbabue & $-3,70$ & n.d. & $-3,70$ & $-3,84$ & $-3,83$ & $-3,78$ & $-3,62$ & $-3,57$ \\
\hline América Latina & 2,81 & 2,20 & 2,15 & 2,06 & 1,92 & 2,04 & 2,48 & 2,60 \\
\hline Argentina & 11,79 & 11,80 & 11,80 & 11,83 & 11,79 & 11,79 & 11,79 & 11,80 \\
\hline Bolivia & 10,33 & n.d. & n.d. & n.d. & n.d. & n.d. & n.d. & n.d. \\
\hline Brasil & 2,07 & 2,04 & 2,03 & 1,93 & 1,94 & 1,99 & 2,15 & 2,20 \\
\hline Colombia & $-0,20$ & $-0,80$ & $-0,89$ & $-0,94$ & $-0,87$ & $-0,59$ & 0,13 & 0,34 \\
\hline Costa Rica & 0,73 & 0,87 & 0,67 & 0,69 & 0,47 & 0,58 & 0,88 & 0,97 \\
\hline Ecuador & $-0,58$ & $-0,56$ & $-0,62$ & $-0,56$ & $-0,60$ & $-0,59$ & $-0,56$ & $-0,55$ \\
\hline El Salvador & 3,02 & 2,77 & 3,03 & 2,48 & 2,51 & 2,29 & 3,61 & 3,96 \\
\hline Guatemala & $-3,78$ & $-3,68$ & $-3,75$ & $-4,10$ & $-4,33$ & $-4,10$ & $-3,49$ & $-3,31$ \\
\hline Jamaica & 2,51 & 0,46 & 2,52 & 2,50 & 2,10 & 2,27 & 2,72 & 2,85 \\
\hline México & 1,62 & 1,48 & 1,46 & 1,31 & 1,25 & 1,40 & 1,81 & 1,93 \\
\hline Panamá & 1,30 & 0,98 & 1,22 & 1,01 & 0,94 & 1,09 & 1,49 & 1,61 \\
\hline Perú & 14,11 & 14,11 & 14,11 & 14,11 & 14,11 & 14,11 & 14,11 & 14,12 \\
\hline Rep. Dominicana & $-0,11$ & $-0,53$ & $-0,07$ & $-0,56$ & $-1,04$ & $-0,63$ & 0,33 & 0,60 \\
\hline San Vicente & $-0,60$ & n.d. & $-0,60$ & $-0,74$ & $-1,01$ & $-0,84$ & $-0,36$ & $-0,21$ \\
\hline Venezuela & $-0,11$ & $-0,39$ & $-0,77$ & $-0,13$ & $-0,38$ & $-0,27$ & 0,03 & 0,13 \\
\hline Asia y Europa emergente & $-1,23$ & $-1,32$ & $-1,23$ & $-1,37$ & $-2,12$ & $-1,70$ & $-0,86$ & $-0,54$ \\
\hline Filipinas & 0,14 & $-0,05$ & 0,24 & $-0,23$ & $-0,24$ & $-0,08$ & 0,34 & 0,47 \\
\hline Fiyi & $-0,45$ & $-0,55$ & $-0,45$ & $-0,55$ & $-1,05$ & $-0,79$ & $-0,17$ & 1,13 \\
\hline India & $-0,93$ & $-1,03$ & $-0,94$ & $-0,96$ & $-1,92$ & $-1,48$ & $-0,47$ & $-0,20$ \\
\hline Indonesia & $-1,09$ & $-0,99$ & $-1,14$ & $-1,45$ & $-1,84$ & $-1,51$ & $-0,74$ & $-0,53$ \\
\hline Malasia & $-4,20$ & $-4,18$ & $-4,22$ & $-4,50$ & $-6,55$ & $-5,42$ & $-3,30$ & $-2,82$ \\
\hline Pakistán & $-1,15$ & $-1,34$ & $-1,12$ & $-1,05$ & $-1,40$ & $-1,30$ & $-1,02$ & $-0,93$ \\
\hline Samoa & $-0,02$ & $-0,02$ & $-0,02$ & $-0,02$ & $-0,02$ & $-0,02$ & $-0,02$ & $-0,02$ \\
\hline Serbia & $-2,85$ & $-2,87$ & $-2,91$ & $-2,80$ & $-3,05$ & $-2,97$ & $-2,74$ & $-2,67$ \\
\hline Tailandia & $-3,42$ & $-3,45$ & $-3,42$ & $-3,48$ & $-6,20$ & $-4,78$ & $-2,48$ & $-1,98$ \\
\hline Turquía & 1,66 & 1,27 & 1,67 & 1,39 & 1,05 & 1,31 & 1,98 & 2,17 \\
\hline Oriente Medio y N. África & $-0,07$ & 0,01 & $-0,21$ & $-0,35$ & $-0,76$ & $-0,51$ & 0,11 & 0,28 \\
\hline Argelia & 0,47 & n.d. & n.d. & n.d. & n.d. & n.d. & n.d. & n.d. \\
\hline Egipto & $-0,11$ & $-0,15$ & $-0,12$ & $-0,11$ & $-0,20$ & $-0,16$ & $-0,05$ & $-0,02$ \\
\hline Jordania & $-1,19$ & $-1,22$ & $-1,17$ & $-1,19$ & $-1,31$ & $-1,26$ & $-1,12$ & $-1,08$ \\
\hline Líbano & 1,59 & 1,80 & 1,65 & 1,23 & $-0,45$ & 0,43 & 2,60 & 3,20 \\
\hline Marruecos & $-0,36$ & $-0,39$ & $-0,36$ & $-0,37$ & $-0,49$ & $-0,44$ & $-0,29$ & $-0,24$ \\
\hline Túnez & $-0,85$ & n.d. & $-1,04$ & $-1,32$ & $-1,33$ & $-1,13$ & $-0,59$ & $-0,44$ \\
\hline
\end{tabular}


spreads permanece casi inalterado: el coeficiente de correlación de ranking entre los spreads originales y las simulaciones de las columnas (2) y (3) es de 0,98 y 0,99, respectivamente. Asimismo, el porcentaje de países con spreads mayores a cero en estas dos simulaciones también se mantiene casi inalterado (33\% y 35\%, respectivamente). Finalmente, en las columnas (4) a (8) se analiza la sensibilidad de los resultados ante modificaciones en el precio final. Como se puede ver, los resultados no son muy sensibles ante modificaciones en el precio final. El rango de spreads varía de $-0,20 \%$ cuando se le descuenta un 25 por ciento a $0,70 \%$ cuando se le incrementa un 25 por ciento, mientras que la proporción de países con spreads positivos va del $29 \%$ al $48 \%$. Asimismo, el ranking entre países permanece en gran medida inalterado: ninguna de las correlaciones de Spearman entre los spreads originales y las simulaciones es inferior a 0,96. Un punto final a notar es que Argentina y Perú son los únicos dos países outliers en todos los ejercicios de robustez.

Intuitivamente, los resultados son tan robustos debido a que al momento de calcular los retornos, las transferencias netas a lo largo del periodo son más relevantes que la valuación del stock final de deuda, ya que dicho stock se descuenta una gran cantidad de periodos y su valor presente termina teniendo una relevancia de segundo orden. En definitiva, el resultado de un spread de largo plazo cercano a cero y la existencia de países con spreads atípicamente altos son robustos a la muestra de países empleada y al método de valuación del stock de deuda en el periodo final, y dichos resultados no son muy sensibles ante variaciones en el precio de mercado de la deuda.

\subsection{Extensiones}

En esta subsección se realizan dos ejercicios adicionales. Primero se analiza la evolución en el tiempo de los spreads ex-post y posteriormente, se comparan los retornos de la deuda soberana con los retornos de una inversión riesgosa.

Para analizar cómo ha sido la evolución de los spreads ex post, en el Cuadro 3 se muestran los spreads a lo largo del tiempo durante tres subperiodos distintos: 1970-1990, 1991-2000 y 2001-2012. Es importante notar que los spreads para dichos subperiodos incluyen submuestras de países ligeramente distintas, de acuerdo a la 
disponibilidad de precios en el mercado secundario en los años intermedios.

Cuadro 3. Spreads ex-post por periodos. (en \%)

\begin{tabular}{|c|c|c|c|}
\hline & $\begin{array}{c}1970-1990 \\
(1)\end{array}$ & $\begin{array}{c}1991-2000 \\
(2)\end{array}$ & $\begin{array}{c}2001-2012 \\
(3)\end{array}$ \\
\hline Todos & $-3,41$ & $-0,40$ & 1,30 \\
\hline África al Sur del Sahara & $-10,77$ & $-2,24$ & 6,41 \\
\hline Congo & $-13,13$ & n.d. & n.d. \\
\hline Costa de Marfil & $-8,31$ & $-5,34$ & $-2,17$ \\
\hline Nigeria & $-10,86$ & 0,86 & 14,99 \\
\hline América Latina & $-0,47$ & 0,27 & 2,10 \\
\hline Argentina & 10,31 & 0,97 & $-7,33$ \\
\hline Brasil & $-1,01$ & 3,54 & 4,19 \\
\hline Colombia & $-3,35$ & $-4,06$ & 5,19 \\
\hline Ecuador & $-12,68$ & $-1,29$ & 2,23 \\
\hline México & $-0,25$ & $-0,35$ & 3,51 \\
\hline Panamá & $-6,72$ & 3,08 & 3,48 \\
\hline Perú & 13,12 & 3,41 & 3,47 \\
\hline Venezuela & $-3,17$ & $-3,12$ & 2,04 \\
\hline Asia y Europa emergente & $-2,85$ & $-1,17$ & 0,12 \\
\hline Filipinas & $-6,98$ & $-3,40$ & 6,74 \\
\hline Indonesia & $-0,42$ & $-0,68$ & $-0,35$ \\
\hline Malasia & $-3,61$ & $-1,03$ & $-3,13$ \\
\hline Tailandia & $-2,77$ & $-1,08$ & $-4,21$ \\
\hline Turquía & $-0,45$ & 0,36 & 1,56 \\
\hline Oriente Medio y N. de África & $-5,55$ & 0,64 & $-2,26$ \\
\hline Egipto & $-5,57$ & n.d. & $-2,41$ \\
\hline Jordania & n.d. & 1,08 & $-1,50$ \\
\hline Marruecos & $-5,53$ & 0,20 & $-2,88$ \\
\hline
\end{tabular}

La media de los spreads ha seguido un patrón ascendente a lo largo de las últimas cuatro décadas: durante el periodo 1970-1990 la media de los spreads fue notablemente negativa $(-3,4 \%)$ y sólo el 11 por ciento de los países tuvo spreads 
positivos, lo cual es en parte consecuencia de las crisis de deuda de los ochenta. Durante los noventa los spreads medios siguieron siendo negativos $(-0,4 \%)$, pero superiores a los de las décadas anteriores, mientras que la proporción de países con spreads positivos ascendió al 47 por ciento. Finalmente, durante los 2000 los spreads medios cambian de signo para volverse positivos (1,3\%) y el porcentaje de países con spreads mayores a cero se incrementó al 55 por ciento. Con respecto a la volatilidad de los spreads, la distribución de los retornos de los noventa presenta la mayor varianza y el mayor rango de variabilidad, mientras que los 2000 fue la década con menor volatilidad. Es interesante notar que ningún país fue un outlier de manera recurrente en todos los subperiodos, sino que el grupo de países con spreads atípicos varía década a década.

Para comparar los retornos de la deuda soberana con los de otra inversión riesgosa se utiliza el retorno del índice Standard \& Poor's 500 (S\&P 500), el cual se basa en la capitalización bursátil de 500 empresas que cotizan en las principales bolsas de valores de Estados Unidos. Estos spreads se ilustran en el Gráfico 4.

Gráfico 4. Spreads ex-post con respecto al índice S\&P 500. 1970-2012

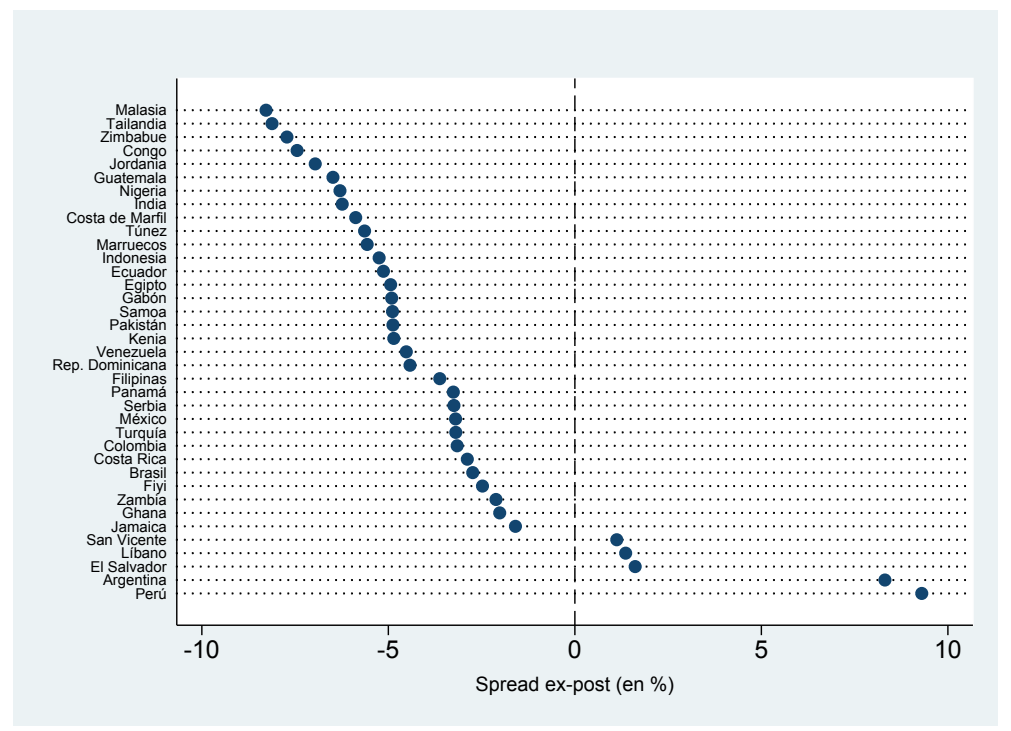

Fuente: elaboración propia en base a datos del Banco Mundial.

Como se puede observar visualmente, en este caso la mayoría de los spreads $(86,5 \%)$ son negativos, sólo cinco países (cuatro de ellos de América Latina y el 
Caribe), tuvieron spreads ex-post positivos. Tanto el spread medio como el mediano tuvieron signos negativos $(-3,5 \%$ y $-4,5 \%$, respectivamente). De manera similar, todos las regiones tuvieron spreads ex-post negativos, aunque con desempeños heterogéneos: el mejor fue el de América Latina $(-1,2 \%)$ y el peor el del Sur de Asia (-5,6\%). Asimismo, ninguno de los grupos de ingreso tuvo spreads positivos, siendo el grupo de países de bajos ingresos aquel con peor desempeño (-6,3\%). Es interesante notar que los países que son outliers en la distribución con respecto a la inversión no riesgosa siguen siendo outliers en la distribución de spreads con respecto a la inversión riesgosa (Malasia del lado negativo de la distribución y Argentina y Perú del lado positivo). Finalmente, el patrón temporal de spreads sigue una evolución ascendente al igual que en el caso de las letras del tesoro, pasando de $-7,2 \%$ durante el periodo 1970-1990, a -0,4\% durante los noventas hasta volverse positivo durante los $2000(1,3 \%)$.

\subsection{Spreads ex-post y percepciones del mercado}

¿Qué nos dicen los spreads ex-post sobre las percepciones del mercado? ¿Cuál es la relación entre los spreads ex-post y las variables que usualmente se consideran relevantes para determinar la rentabilidad de la deuda pública? Para responder a estas preguntas, se analiza la correlación entre los spreads ex-post, un grupo de variables que reflejan las percepciones del mercado sobre los países, y un conjunto de variables típicamente asociadas con los retornos de la deuda soberana. Es importante notar que el objetivo de esta subsección no es hallar los determinantes de los spreads ni inferir causalidad, sino simplemente investigar sobre la existencia de posibles asociaciones entre las variables.

Con respecto al grupo de variables que capturan las percepciones del mercado se incluye la prima de riesgo de cada país, e índices del estado de derecho, calidad regulatoria, efectividad gubernamental, control de la corrupción, estabilidad política y rendición de cuentas. Con respecto al grupo de variables usualmente asociadas con los retornos se incluye el grado de apertura financiera de cada país, el porcentaje de la deuda en moneda extranjera medio, el número de defaults y la cantidad de años en default a lo largo del periodo, el monto promedio de deuda en default (en $\%$ del INB), el monto promedio de reestructuración (en \% del INB) y el recorte 
promedio de las reestructuraciones. La definición de las variables y su fuente de información se encuentra en el Apéndice E. En los Gráficos E.1 y E.2 del mismo Apéndice también se ofrece una inspección visual de la correlación de los distintos grupos de variables con los spreads ex-post. Los principales resultados se resumen en el Cuadro 4.

Cuadro 4. Variables asociadas con los spreads ex-post

\begin{tabular}{lccccc}
\hline & $\begin{array}{c}\text { Corr. lineal } \\
(1)\end{array}$ & $\begin{array}{c}\text { Corr. ranking } \\
(2)\end{array}$ & $\begin{array}{c}\mathrm{MCO} \\
(3)\end{array}$ & $\begin{array}{c}\mathrm{R}^{2} \\
(4)\end{array}$ & $\begin{array}{c}\text { Obs. } \\
(5)\end{array}$ \\
\hline \hline Percepciones del mercado & & & & & \\
Prima de riesgo (rating calificadoras) & 0,09 & 0,04 & 0,15 & 0,01 & 34 \\
Prima de riesgo (credit default swaps) & 0,40 & 0,07 & 0,53 & 0,16 & 17 \\
Estado de derecho & 0,04 & 0,22 & 0,00 & 0,00 & 37 \\
Calidad regulatoria & 0,26 & 0,35 & $0.01^{*}$ & 0,07 & 37 \\
Efectividad gubernamental & 0,11 & 0,27 & 0,01 & 0,01 & 37 \\
Control de la Corrupción & 0,14 & 0,28 & 0,01 & 0,02 & 37 \\
Estabilidad política & 0,11 & 0,29 & 0,01 & 0,01 & 37 \\
Rendición de Cuentas & 0,34 & 0,53 & $0.02 * * *$ & 0,12 & 37 \\
& & & & & \\
Gestión de la deuda soberana & & & & & \\
Apertura de la cuenta de capital & 0,13 & 0,14 & 0,00 & 0,02 & 36 \\
Deuda en moneda extranjera & 0,04 & $-0,04$ & 0,06 & 0,00 & 37 \\
Número de defaults & 0,33 & 0,31 & $0.01^{*}$ & 0,11 & 37 \\
Monto en default medio (\% INB) & $-0,04$ & 0,04 & $-0,01$ & 0,00 & 36 \\
Años en default en la muestra & 0,14 & 0,10 & 0,00 & 0,02 & 37 \\
Reestructuración media (\% INB) & $-0,11$ & $-0,23$ & $-0,05$ & 0,01 & 22 \\
Recorte medio (\% de la deuda) & $-0,26$ & $-0,41$ & $-0,05$ & 0,07 & 22 \\
\hline
\end{tabular}

Nota: la columna (1) muestra el coeficiente de correlación lineal de Pearson entre los spreads ex-post y las distintas variables; la columna (2) muestra el coeficiente de correlación de ranking de Spearman; la columna (3) el coeficiente de una regresión lineal por Mínimos Cuadrados Ordinarios sin controles en donde la variable dependiente son los spreads ex-post. $* * *, * * \mathrm{y} *$ denotan significatividad al $10 \%, 5 \%$ y $1 \%$, respectivamente, calculadas empleando errores estándar robustos. La columna (4) muestra el R-cuadrado de dicha regresión y la última columna el número de observaciones disponibles en cada una de las variables. 
Como se puede observar en el Cuadro 4, las correlaciones entre el grupo de variables que intentan capturar las percepciones del mercado y los spreads ex-post son bastante débiles (columnas 1 y 2). La percepción del riesgo de cada país por parte del mercado se asocia de manera positiva con los spreads ex-post. Una posible interpretación de estos coeficientes es que los países que ex-post brindaron mayores retornos son aquellos que los mercados consideran más riesgosos. Las únicas dos variables estadísticamente distintas de cero a los niveles usuales de significatividad en las regresiones son la calidad regulatoria del país y el índice de rendición de cuentas, los cuales ambos se asocian de manera positiva con los spreads ex-post (columna 3). Por su parte, el poder explicativo de este conjunto de variables es muy bajo (columna 4). La variable con mayor R-cuadrado (el riesgo país medido mediante los credit default swaps) tan sólo puede explicar el 16\% de la variabilidad de los retornos ex-post. En otras palabras, no hay una relación robusta entre cuan "amigable al mercado" se piensa que son los países y los spreads ex-post.

Los resultados son cuantitativamente similares con respecto al segundo conjunto de variables. Las variables con asociaciones más fuertes son el número de defaults y la cantidad de años en default de manera positiva, y el monto de deuda reestructurado y su "recorte" asociado promedio (columnas 1 y 2). La variable con mayor poder explicativo es el número de defaults, el cual se asocia de manera positiva y significativa con los spreads ex-post (columnas 3 y 4). Una posible interpretación de este coeficiente está en línea con los resultados hallados en el párrafo anterior: los países que tuvieron un mayor número de defaults en el agregado fueron los que brindaron mayores spreads ex-post. Esto se debe a que el "premio al riesgo" con el que compensaron a los acreedores durante los periodos en los que sí pagaron su deuda más que compensó las perdidas asociadas a los defaults y posteriores reestructuraciones. En definitiva, la asociación entre los spreads ex-post y las variables que típicamente se consideran como determinantes de ellos también resulta débil y su poder explicativo es bajo. 


\section{Reflexiones finales}

En el libro "El descubrimiento de la India" el ex-primer ministro indio Jawāharlāl Nehru nota que "la historia la escriben casi siempre los vencedores". De manera similar, la historia financiera pareciera estar escrita por los acreedores. Cuando ocurre una crisis soberana, a menudo se asume que el único culpable es del deudor. Sin embargo, los contratos de deuda invariablemente tienen dos partes. El fracaso de un préstamo representa errores de cálculo de ambos lados de la transacción. En los estados favorables de la naturaleza, los países honran sus obligaciones y pagan los servicios de su deuda. Sin embargo, hay circunstancias en las que para los países es económica y socialmente inviable pagar sus deudas. En dichas situaciones es necesario llevar a cabo una reestructuración, disminuyendo su valor para que el país pueda recuperar la capacidad de crecimiento.

Justamente por la posibilidad de que esas circunstancias ocurran es que los países emergentes pagan una mayor tasa de interés por sus deudas, de modo de compensar a los acreedores con mayores pagos en los momentos en los que la deuda sí se puede pagar. Invertir en deuda de un país emergente que brinda un "premio por el riesgo" implica que los inversores son conscientes de que en algunos estados de la naturaleza el soberano puede hacer default, e implica apostar a que el retorno del deudor soberano va a ser mayor que el del costo de oportunidad. En circunstancias normales, los acreedores cobran una sobretasa, pero en el caso de que el país tenga un shock negativo y haga default, los acreedores dejan de cobrar hasta que el país reestructure su deuda. Aun en este caso es posible que el retorno de sus acreedores sea positivo e incluso superior al de inversiones alternativas. Esto es justamente lo que se pretendió investigar en este trabajo.

En este trabajo se calculan los spreads ex-post de la deuda pública de una muestra de 37 países emergentes usando información de flujos agregados. El hecho de que se emplee información agregada y no a nivel bono para estimar los retornos limita la interpretación de los resultados encontrados ya que, como se encuentra en el trabajo, los resultados son sensibles a variaciones en el periodo bajo análisis. En particular, la elección del periodo inicial influye en gran medida sobre el ranking de países en términos de "exceso de retornos" y en menor medida sobre la media de los spreads ex-post. Este trabajo puede ser extendido calculando los retornos 
ex-post a nivel bono (así como el costo de oportunidad asociado a cada bono). Dicho ejercicio le daría un mayor alcance a la interpretación de los resultados y ayudaría a corroborar la validez de los resultados de este trabajo.

El principal resultado del trabajo es que durante el periodo 1970-2012, los spreads ex-post fueron, en promedio, positivos y cercanos a cero. También se hallaron los países que brindaron spreads ex-post atípicamente altos durante dicho periodo. Se corroboró la robustez de estos resultados ante un posible sesgo de selección y ante diversos escenarios con respecto al precio de la deuda en el mercado secundario. Desagregando el análisis por décadas se encontró que los spreads han tenido una trayectoria ascendente durante los últimos cuarenta años. También se analizó el retorno de la deuda emergente en comparación con los retornos del índice S\&P 500, encontrando que este brindó retornos mayores que la deuda soberana. Finalmente, no se encontró una relación robusta entre variables que indican percepciones sobre "tendencias pro-mercado" de los países y retornos expost, y las variables que típicamente se consideran como determinantes cruciales de los retornos exhibieron un poder explicativo bajo de los spreads. 


\section{Apéndice A. Valuación de deuda soberana}

En esta Apéndice se esboza un modelo de equilibrio general dinámico y estocástico de default soberano con reestructuraciones de deuda, basado en el trabajo seminal de Eaton y Gersovitz (1981) extendido por Yue (2010) con el fin de analizar los contratos de deuda óptimos y el mecanismo de valuación de la deuda soberana cuando hay riesgo de default y no existen mecanismos de auditoría posibles en caso de no pago.

Se considera una economía pequeña y abierta en tiempo discreto, $t=\{0,1,2, \ldots, \infty)$. El gobierno del país es benevolente y maximiza la utilidad intertemporal esperada de los hogares. Los hogares que habitan el país son idénticos y sus preferencias están dadas por la siguiente expresión:

$$
\mathbb{E}_{0} \sum_{t=0}^{\infty} \beta^{t} u\left(c_{t}\right)
$$

en donde $\mathbb{E}_{0}$ denota la expectativa del hogar en el periodo cero, $\beta \in(0,1)$ es el factor de descuento subjetivo, $c_{t}$ el consumo en el periodo $t$ y $u\left(c_{t}\right)$ es la función de utilidad, la cual se supone continua, estrictamente creciente, estrictamente cóncava y que satisface las condiciones de Inada. En cada periodo, el país recibe una dotación estocástica y exógena del único bien de consumo $y_{t}$.

Los inversores internacionales son neutrales al riesgo y poseen información perfecta acerca de la dotación del país y su posesión de activos. Asimismo, pueden prestar o pedir prestado a la tasa de interés internacional libre de riesgo, $r_{t}^{*}$. Los mercados de capitales son incompletos y perfectamente competitivos. El gobierno del país puede prestar o pedir prestado sólo mediante un bono cupón cero que vence en el periodo siguiente a su emisión. El valor nominal de este bono se denota $b_{t+1}$, y especifica el valor a ser pagado durante el periodo siguiente a su emisión. Cuando el gobierno compra bonos $b_{t+1}>0$ y cuando emite deuda $b_{t+1}<0$. Se denota $q\left(b_{t+1}, y_{t}\right)$ al precio de un bono con un valor nominal $b_{t+1}$, emitido por un país que tiene una dotación $y_{t}$. El precio del bono y su retorno se determinan en equilibro.

Los inversores internacionales se comprometen a pagar siempre su deuda. Sin embargo, el soberano puede decidir no pagar sus compromisos y entrar en default. 
Cuando el país hace default sobre su deuda, el valor presente de ésta se reduce a una fracción $\alpha_{t}\left(b_{t}, y_{t}\right)$, que a su vez representa la tasa de recupero de la deuda, es decir, la fracción de la deuda que el soberano paga después de renegociar su deuda. Por lo tanto, la negociación sobre la tasa de recupero de la deuda es equivalente a la negociación sobre la reducción de la deuda. En el modelo se determinará un valor óptimo de reducción de deuda en el proceso de reestructuración, el cual maximiza el beneficio agregado de la negociación para ambas partes. A medida que cantidad de deuda en default aumenta, la tasa de recupero de la deuda disminuye. En otras palabras, la tasa de recupero de la deuda será menor para crisis de deuda más severas.

Si el país entra en default, éste no realiza ningún pago de deuda durante dicho periodo y su historial de crédito se deteriora. Los países con un historial crediticio deteriorado y deuda pendiente de pago son excluidos de los mercados internacionales de crédito.

Una vez producida la cesación de pagos, los acreedores pueden coordinar colectivamente y negociar con el país una reducción de la deuda en un juego de Nash. La tasa de recupero de la deuda se determina en la negociación posterior al default. De acuerdo al resultado de la negociación, el valor presente de la deuda en default se reduce a una fracción del total de la deuda impaga. El país sale del default cuando paga su deuda morosa. Una vez que dicha deuda es pagada, su historial crediticio se limpia y recupera el acceso al mercado de capitales. Si la negociación falla, los acreedores internacionales pierden su inversión y el país queda excluido de manera indefinida de los mercados financieros.

En el equilibrio recursivo se resuelve simultáneamente el problema de maximización de la utilidad esperada por parte del gobierno soberano, el juego de Nash en el cual se determinan las condiciones de la reestructuración de la deuda y la maximización del beneficio esperado por parte de los inversores extranjeros. En este trabajo sólo se analiza el último de dichos problemas (cf. Yue (2010) para la caracterización completa del equilibrio).

Tomando el precio del bono como dado, los inversores extranjeros eligen el monto de deuda $b_{t+1}$ que maximiza su beneficio esperado, $\pi_{t}\left(b_{t+1}, y_{t}\right)$. Dicho beneficio viene dado por: 


$$
\pi_{t}\left(b_{t+1}, y_{t}\right)= \begin{cases}\left(q_{t}\left(b_{t+1}, y_{t}\right)-\frac{1}{1+r_{t}^{*}}\right) b_{t+1} & \text { si } b_{t+1} \geq 0 \\ \left(q_{t}\left(b_{t+1}, y_{t}\right)-\frac{\left[1-p_{t}\left(b_{t+1}, y_{t}\right)\right]+p_{t}\left(b_{t+1}, y_{t}\right) \phi_{t}\left(b^{\prime}, y_{t}\right)}{1+r_{t}^{*}}\right) b_{t+1} & \text { si } b_{t+1}<0\end{cases}
$$

en donde $\phi_{t}\left(b_{t+1}, y_{t}\right)$ es la tasa de recupero esperada condicional a que el soberano haya hecho default y $p\left(b_{t+1}, y_{t}\right)$ es la probabilidad esperada de que un país con nivel de deuda $b_{t+1}$ y dotación $y_{t}$ entre en default. A medida que el stock de deuda del país aumenta, el incentivo a cesar sus pagos es mayor, y por lo tanto la probabilidad de que haga default se incrementa. Asimismo, en estados desfavorables de la naturaleza -cuando $y_{t}$ es relativamente bajo- la utilidad marginal del consumo es mayor, por lo que el soberano tiene un mayor incentivo a hacer default y la probabilidad de default se incrementa. En otras palabras, en el caso un shock negativo, el soberano puede "aceptarlo" o entrar en default. El incentivo a incumplir los términos formales del contrato está limitado por los costos asociados con la ruptura; sin embargo, si es necesaria una caída del consumo demasiado intensa para pagar los servicios de la deuda, el shock puede causar la suspensión de los pagos contractuales.

Asumiendo competencia perfecta en el mercado internacional de crédito, el beneficio esperado de los inversores extranjeros en equilibrio es nulo, por lo tanto:

$$
q_{t}\left(b_{t+1}, y_{t}\right)= \begin{cases}\frac{1}{1+r_{t}^{*}} & \text { si } b_{t+1} \geq 0 \\ \frac{1-p_{t}\left(b_{t+1}, y_{t}\right)}{1+r_{t}^{*}}+\frac{p_{t}\left(b_{t+1}, y_{t}\right) \phi_{t}\left(b_{t+1}, y_{t}\right)}{1+r_{t}^{*}} & \text { si } b_{t+1}<0\end{cases}
$$

Cuando el soberano les presta a los inversores extranjeros, el precio del bono soberano es igual al precio del bono libre de riesgo, y por lo tanto los retornos de ambos bonos son iguales. Cuando el país le pide prestado a los acreedores extranjeros, éstos se exponen al riesgo de que el soberano incumpla sus promesas y al riesgo de que posteriormente falle la negociación con el soberano y no se reestructure la deuda en default. El bono soberano es valuado de forma tal de compensar a los acreedores por cargar con ambos riesgos. Como el precio del bono soberano depende de la probabilidad de que el soberano haga default y dicha 
probabilidad depende de la cantidad de deuda pendiente de pago y del shock de dotación, el precio del bono también es una función de ambas variables.

Dado que $p_{t}\left(b_{t+1}, y_{t}\right) \in[0,1]$ y que $\phi_{t}\left(b_{t+1}, y_{t}\right) \in[0,1]$, el precio del bono $q_{t}\left(b_{t+1}, y_{t}\right)$ se sitúa en el intervalo $\left[0, \frac{1}{\left(1+r_{t}^{*}\right)}\right]$. La tasa de interés del bono riesgoso, $r_{t}\left(b_{t+1}, y_{t}\right)=\frac{1}{q_{t}\left(b_{t+1}, y_{t}\right)}-1$, está acotada inferiormente por la tasa de interés libre de riesgo. Reemplazando la expresión del precio del bono riesgoso en la ecuación (4) se llega a la condición de arbitraje entre tasas:

$$
1+r_{t}^{*}=\left[1-p_{t}\left(b_{t+1}, y_{t}\right)\right]\left(1+r_{t}\left(b_{t+1}, y_{t}\right)\right)+p_{t}\left(b_{t+1}, y_{t}\right) \phi_{t}\left(b_{t+1}, y_{t}\right)\left(1+r_{t}\left(b_{t+1}, y_{t}\right)\right)
$$

La ecuación (5) indica que el riesgo de default tiene un efecto de primer orden sobre la valuación del bono riesgoso y, por lo tanto, sobre la tasa de interés que paga el soberano. A medida que aumenta la probabilidad de que el soberano haga default, mayor será la sobretasa que requieran los acreedores para comprar bonos riesgosos. Por su parte, la tasa de recupero esperada afecta el precio del bono a través de su efecto combinado con el riesgo de default.

La tasa de interés de los bonos soberanos también puede depender de las características de los inversores, y no sólo de los fundamentales de la economía. Si se consideran inversores aversos al riesgo, la rentabilidad de los bonos soberanos dependerá del grado de aversión al riesgo de los acreedores. Entre más aversos al riesgo sean los inversores, la tasa de interés de los bonos soberanos será mayor (cf. Lizarazo, 2013). Asimismo, si se consideran inversores aversos a la incertidumbre, es decir, que toman decisiones robustas a la distribución de probabilidad del modelo que rige la dotación del soberano, la tasa de interés de los bonos públicos también dependerá del grado de aversión a la incertidumbre de los acreedores. Entre más incertidumbre tengan los inversores acerca del verdadero modelo que rige la economía, mayor será la sobretasa que requieren para demandar bonos riesgosos (cf. Pouzo y Presno, 2013). 


\section{Apéndice B. Definiciones conceptuales}

En este apartado se ofrecen las definiciones de los principales conceptos empleados a lo largo del trabajo.

\section{Amortizaciones de capital}

Las amortizaciones de capital son los montos de capital (reembolsos) efectivamente pagados por el soberano en divisas, bienes o servicios en el año especificado.

\section{Capitalización de intereses}

Interés capitalizado es el interés que se convirtió en parte del saldo de la deuda debido a una operación de reprogramación. Interés reprogramado es el importe de los intereses devengados o vencidos que fue reprogramado en un año determinado.

\section{Composición por monedas del stock de deuda}

La composición por monedas del stock de deuda está dada por el porcentaje de deuda soberana contraída en moneda extranjera para los países de bajos y medianos ingresos. Esta información se encuentra desagregada para las siguientes divisas: marco alemán, euro, franco francés, yen japonés, libra esterlina, franco suizo, dólar estadounidense y derechos especiales de giro.

\section{Condonaciones de la deuda}

La condonación de la deuda muestra el cambio en el saldo de la deuda debido a la condonación de la deuda. Se obtiene restando la deuda perdonada y las reducción en el stock de deuda de las recompras de deuda.

\section{Desembolso}

El desembolso es el monto otorgado por parte de los acreedores cuando el soberano asume un nuevo préstamo durante el año especificado.

\section{Deuda soberana con acreedores privados}

La deuda soberana con acreedores privados se compone de tres instrumentos distintos: bonos, préstamos bancarios y financiación por parte de otros acreedores 
privados. Se incluye los bonos que se emiten ya sea públicamente o de manera privada con garantía púbica; los préstamos de bancos comerciales privados y otras instituciones financieras privadas; y la financiación de otros acreedores privados, compuesta por los créditos procedentes de proveedores de bienes como exportadores o fabricantes y los créditos bancarios garantizados por una agencia de crédito a la exportación.

\section{Flujos netos}

Los flujos netos (o financiamiento neto o desembolsos netos) recibidos por el prestatario durante el año son los desembolsos menos las amortizaciones de capital.

\section{Intereses atrasados}

El interés atrasado sobre la deuda soberana se define como el pago de intereses vencidos y no pagados, de forma acumulada.

\section{Pago de intereses}

El pago de intereses son las cantidades reales de interés pagados por el soberano en divisas, bienes o servicios en el año especificado.

\section{Recompra de deuda}

Recompra de deuda es el rescate por parte del deudor de su propia deuda, con descuento o a la par. En el caso de recompra de deuda soberana, el valor nominal de la deuda rescatada se registra como una disminución en el saldo del stock de deuda pendiente de pago, y el importe efectivo recibido por los acreedores se registrarán como amortización de capital.

\section{Servicios de la deuda}

Los servicios de la deuda son la suma del pago de intereses y las amortizaciones de capital interés pagados por el soberano en divisas, bienes o servicios en el año especificado. 


\section{Stock de deuda soberana}

El stock de deuda soberana es la deuda contraída con acreedores no residentes del país, reembolsable en divisas, bienes o servicios. La deuda soberana es la suma de la deuda pública y de la deuda con garantía pública, ambas de largo plazo. La deuda pública es una obligación externa de un deudor público, incluyendo el gobierno nacional, una subdivisión política, y los organismos públicos autónomos. La deuda con garantía pública es una obligación externa de un deudor privado que está garantizado para el reembolso por una entidad pública. La deuda a largo plazo se define como deuda con un vencimiento original o extendido de más de un año y que se le debe a no residentes por los residentes de una economía y reembolsable en divisas, bienes o servicios.

\section{Transferencias netas}

Las transferencias netas son los flujos netos menos los pagos de intereses durante el año; es decir, los desembolsos menos los servicios de la deuda. Transferencias negativas indican transferencias netas realizadas por el soberano al acreedor durante el año y viceversa. 


\section{Apéndice C. Características de la muestra de países}

\begin{tabular}{|c|c|c|c|c|c|c|}
\hline \multirow{2}{*}{ País } & \multirow{2}{*}{$\begin{array}{l}\text { Nivel de } \\
\text { ingreso }\end{array}$} & \multirow{2}{*}{$\begin{array}{c}\text { Defaults } \\
\text { soberanos }\end{array}$} & \multicolumn{2}{|c|}{ Deuda en default (\% INB) } & \multicolumn{2}{|c|}{ Recorte (\% de la deuda) } \\
\hline & & & Privada & Total & Media & Mediana \\
\hline \multicolumn{7}{|c|}{ África al Sur del Sahara } \\
\hline Congo & Medio-bajos & 1 & 23.9 & 62.7 & 66.5 & 66.5 \\
\hline Costa de Marfil & Medio-bajos & 2 & 0.0 & 34.4 & 41.4 & 55.2 \\
\hline Gabón & Medio-bajos & 2 & 0.2 & 7.3 & 12.1 & 12.1 \\
\hline Ghana & Medio-altos & 0 & - & - & - & - \\
\hline Kenia & Bajos & 0 & - & - & - & - \\
\hline Nigeria & Medio-bajos & 1 & 2.0 & 20.6 & 21.6 & 24.7 \\
\hline Rep. Dem. Congo & Bajos & 1 & 5.9 & 45.6 & 35.4 & 35.4 \\
\hline Zambia & Medio-bajos & 1 & 3.9 & 45.1 & 89.0 & 89.0 \\
\hline Zimbabue & Bajos & 0 & - & - & - & - \\
\hline \multicolumn{7}{|l|}{ América Latina } \\
\hline Argentina & Medio-altos & 2 & n.d. & 13.6 & 40.3 & 31.4 \\
\hline Bolivia & Medio-bajos & 1 & 0.7 & 9.7 & 84.6 & 84.6 \\
\hline Brasil & Medio-altos & 1 & 0.3 & 5.3 & 14.3 & 18.8 \\
\hline Colombia & Medio-altos & 0 & - & - & - & - \\
\hline Costa Rica & Medio-altos & 1 & 0.0 & 6.6 & 49.0 & 39.4 \\
\hline Ecuador & Medio-altos & 3 & 0.1 & 14.9 & 29.3 & 26.9 \\
\hline El Salvador & Medio-bajos & 0 & - & - & - & - \\
\hline Guatemala & Medio-bajos & 0 & - & - & - & - \\
\hline Jamaica & Medio-altos & 2 & 0.6 & 9.8 & 21.1 & 18.1 \\
\hline México & Medio-altos & 1 & 0.0 & 5.5 & 21.7 & 18.1 \\
\hline Panamá & Medio-altos & 1 & 0.1 & 19.2 & 20.7 & 15.1 \\
\hline Perú & Medio-altos & 1 & 0.0 & 14.5 & 14.6 & 0.9 \\
\hline Rep. Dominicana & Medio-altos & 2 & 0.2 & 6.6 & 36.1 & 49.9 \\
\hline San Vicente & Medio-altos & 0 & - & - & - & - \\
\hline Venezuela & Medio-altos & 1 & 0.2 & 8.0 & 17.0 & 9.9 \\
\hline \multicolumn{7}{|c|}{ Asia y Europa emergente } \\
\hline Filipinas & Medio-bajos & 1 & 0.2 & 6.0 & 31.6 & 34.0 \\
\hline Fiyi & Medio-altos & 0 & - & - & - & - \\
\hline India & Medio-bajos & 0 & - & - & - & - \\
\hline Indonesia & Medio-bajos & 1 & 1.0 & 1.5 & - & - \\
\hline Malasia & Medio-altos & 0 & - & - & - & - \\
\hline Pakistán & Medio-bajos & 0 & 0.0 & 0.0 & 0.0 & 0.0 \\
\hline Samoa & Medio-bajos & 0 & - & - & - & - \\
\hline Serbia & Medio-altos & 0 & - & - & - & - \\
\hline Tailandia & Medio-altos & 0 & - & - & - & - \\
\hline Turquía & Medio-altos & 1 & 0.0 & 0.5 & 15.5 & 17.0 \\
\hline \multicolumn{7}{|c|}{ Oriente Medio y N. de África } \\
\hline Argelia & Medio-altos & 0 & - & - & - & - \\
\hline Egipto & Medio-bajos & 1 & 0.5 & 5.8 & n.d. & n.d. \\
\hline Jordania & Medio-altos & 1 & 1.8 & 5.9 & 54.6 & 54.6 \\
\hline Líbano & Medio-altos & 0 & - & - & - & - \\
\hline Marruecos & Medio-bajos & 1 & 0.2 & 2.5 & 28.4 & 23.5 \\
\hline Túnez & Medio-altos & 0 & - & - & - & - \\
\hline
\end{tabular}




\section{Apéndice D. Aspectos metodológicos}

Computar los spreads de retornos ex-post de la deuda soberana de los países emergentes en base a los flujos de capitales observados es un desafío complejo que requiere de numerosos ajustes y algunos supuestos. En este Apéndice Metodológico se detallan los problemas conceptuales para calcular las tasas de retorno y los ajustes llevados a cabo para resolverlos.

\section{D.1 Ajuste en las transferencias netas}

El primer gran ajuste que se llevó a cabo fue para corregir los flujos de transferencias netas publicados por el Banco Mundial. El problema con la información proporcionada por la base de datos IDS sobre transferencias netas es que brinda retornos erróneos en el caso de que haya operaciones de reestructuración o consolidación de deuda que involucren intercambios de instrumentos entre distintas clases de activos. Cuando sucede esto, la IDS registra un cambio en el stock en una categoría de deuda, pero la contrapartida es registrada en una categoría distinta, lo cual lleva a errores en la medición de la tasa de retorno. Algunos ejemplos:

- En el caso de un canje de deuda pública por acciones de empresas, la IDS registra una disminución en el stock de deuda -así como en los subsecuentes servicios de dicha deuda- lo cual no está asociado a ningún pago de deuda. Desde la perspectiva del inversor, recibir las acciones constituye un derecho a recibir un nuevo flujo de pagos. Ignorar este derecho llevaría a subestimar el retorno sobre la deuda.

- En el caso de consolidación de deuda de corto plazo a largo plazo - tal como ocurrió durante la crisis de la deuda de los 80-, la IDS registra una disminución en el stock de deuda de corto plazo, lo cual nuevamente no está asociada a ningún pago de deuda, mientras que la deuda de largo plazo (así como los servicios de la deuda de largo plazo) aumentan sin que haya ningún desembolso nuevo por parte de los acreedores. Por lo tanto, calcular la tasa de retorno sobre los flujos de deuda de largo plazo llevaría a una sobreestimación de la tasa de retorno de largo plazo, mientras que los flujos de deuda de corto plazo llevarían a una subestimación de los retornos de la deuda de corto plazo. 
Para solucionar este inconveniente, es necesario construir una serie de transferencias netas modificadas que contemple los intercambios de activos entre distintas categorías. Para esto se sigue el enfoque sugerido por Lindert (1989), el cual se basa en la siguiente identidad que relaciona stocks y flujos:

$$
\Delta D_{t}=d_{t}+a_{t}+v_{t}+n_{t}+x_{t}+\epsilon_{t}
$$

En donde $\Delta D$ representa el cambio en el stock de deuda de largo plazo (medido en dólares); $d$ los desembolsos por parte de los acreedores; $a$ los pagos por amortizaciones de capital; $c$ las condonaciones y reducciones de deuda; $v$ los cambios en el valor en dólares de la deuda como consecuencia de movimientos en el tipo de cambio entre la divisa en la cual está denominada la deuda y el dólar; $n$ los intereses y atrasos de intereses capitalizados; $x$ es la variable que denota cambios en el stock de deuda como consecuencia de reestructuraciones que involucraron cambios entre distintos tipos de activos y $\epsilon$ es un residuo atribuible a errores de medición en cualquiera de las categorías de la identidad el cual, en principio, debería ser cero. Con la excepción de $x$ y $\epsilon$, todas las variables de la identidad son observables o pueden ser construidas en base a la información publicada por el Banco Mundial, por lo cual, $x$ es computada como el residuo de dicha ecuación.

\section{D.2 Valuación del stock de deuda soberana a precios de mercado}

El segundo ajuste se llevó a cabo para valuar el stock de deuda soberana a precios de mercado secundario. La corrección consiste en tomar el monto pendiente de pago de deuda total y multiplicarlo por un promedio ponderado de los precios en el mercado secundario. Dicho precio promedio se computa considerando todos instrumentos de deuda para los cuales hay precios disponibles y usando el valor pendiente de pago de cada instrumento como ponderador.

Evaluar el stock de deuda a valores de mercado tiene algunos inconvenientes prácticos. En primer lugar, no hay información disponible sobre precios en el mercado secundario antes del año 1986, lo cual imposibilitaría el cómputo de las tasas de retorno para submuestras que comiencen antes de dicho año. Para 
computar la tasa de retorno del periodo completo (1970-2012), la deuda pendiente de pago en 1970 se valúa a la par. En la medida en la que dicha deuda en la realidad hubiera valido menos, las tasas de retorno computadas pueden estar subestimadas. Sin embargo, cualquier sesgo sería mínimo ya que, por una parte, la cantidad de deuda emergente en posesión de acreedores privados en 1970 era mínima relativa a los flujos de fondos que le siguieron ${ }^{7}$. Adicionalmente, cualquier descuento del mercado hubiera sido pequeño ya que el año 1970 precede a los primeros episodios de dificultades de pago de deuda que involucraron a países emergentes y acreedores privados.

El segundo inconveniente es que los precios disponibles a partir del año 1986 sean representativos de todos los instrumentos de deuda. Si bien esto no conlleva problemas dentro de cada tipo de instrumento -como bonos o préstamos bancarios-, es menos claro que el precio de los bonos se pueda utilizar para valuar los préstamos bancarios y viceversa. La existencia o no de un sesgo dependerá de si los términos de los instrumentos para los cuales no hay precios disponibles son similares a los del resto de los instrumentos, así como del tamaño relativo de estos instrumentos con respecto al total del stock de deuda.

En la práctica, el precio agregado de la deuda se obtuvo de Klingen et al. (2004) para los años previos al 2000 y de Bloomberg para el 2011 y 2012. Para estimar el precio agregado del 2012 se obtuvo información de 16,949 bonos emitidos por los deudores soberanos y para el 2011 información de 15,901 bonos. De este total de bonos, tan sólo una submuestra de 675 y 678 fueron comerciados y, por lo tanto, cuentan con precios de mercado. El precio agregado se calcula como un promedio ponderado del precio de dichos bonos, en donde el ponderador es el valor pendiente por amortizar. En el trabajo se muestra que los resultados son robustos a utilizar información de todos los bonos disponibles, imputándoles un precio en función de sus características observables mediante el método de estratificación.

\footnotetext{
${ }^{7}$ La deuda soberana de los países en vías de desarrollo en manos de acreedores privados se multiplicó casi por veinte entre 1970 y 1982.
} 


\section{Apéndice E. Variables usadas para las correlaciones}

En el Cuadro E.1 se resumen las fuentes de información de las variables que fueron empleadas para correlacionar los spreads ex-post.

Cuadro E.1 Fuentes de información de las variables usadas para las correlaciones

\begin{tabular}{ll}
\hline Variable & Fuente \\
\hline \hline Prima de riesgo & Aswath Damodaran \\
Apertura de la cuenta de capital & Chinn e Ito (2006) \\
Estado de derecho & Worldwide Governance Indicators \\
Calidad regulatoria & Worldwide Governance Indicators \\
Efectividad gubernamental & Worldwide Governance Indicators \\
Control de la Corrupción & Worldwide Governance Indicators \\
Estabilidad política y ausencia de violencia & Worldwide Governance Indicators \\
Voz y rendición de Cuentas & Worldwide Governance Indicators \\
Porcentaje de la deuda en moneda extranjera & Indicadores del desarrollo mundial \\
Número de defaults & Laeven y Valencia (2013) \\
Monto en default medio (en \% INB) & Beers y Nadeau (2014) \\
Reestructuración meda (en \% INB) & Cruces y Trebesch (2013) \\
Años en default en la muestra & Beers y Nadeau (2014) \\
Recorte promedio de cada reestructuración & Cruces y Trebesch (2013) \\
\hline
\end{tabular}

Las primas de riesgo de cada país se obtuvieron de la base de datos de Aswath Damodaran, quien calcula la prima de riesgo utilizando los rating de la calificadora de riesgo Moody's y utilizando el spread implícito en los credit default swaps (CDS) 8 . Esta variable pretende medir el grado de riesgo de un país para los inversionistas extranjeros.

De manera complementaria a las primas de riesgo, se utilizan un conjunto de medidas que pretenden capturar la percepción de los mercados acerca de los países. Dichas medidas son las siguientes:

- Voz y rendición de cuentas: captura la percepción de la medida en que los ciudadanos de un país son capaces de participar en la selección de su gobierno,

\footnotetext{
${ }^{8}$ Los CDS son contratos de seguros que se comercian para protegerse de inversiones riesgosas las inversiones de riesgo. El comprador de un CDS otorga una serie de pagos al vendedor a cambio de asegurar el instrumento financiero subyacente (en este caso, la deuda soberana) en contra de algún evento (en este caso, default por parte del país).
} 
así como la libertad de expresión, la libertad de asociación y la libertad de los medios de comunicación.

- Estabilidad política: captura la percepción de la probabilidad de que haya inestabilidad política y / o la violencia por motivos políticos, incluido el terrorismo.

- Efectividad gubernamental: captura la percepción de la calidad de los servicios públicos, la calidad de la administración pública y el grado de independencia de presiones políticas, la calidad de la formulación y aplicación de políticas, y la credibilidad del compromiso del gobierno para cumplir con tales políticas.

- Calidad regulatoria: captura la percepción de la capacidad del gobierno para formular y aplicar políticas y reglamentaciones acertadas que permitan y promuevan el desarrollo del sector privado.

- Estado de derecho: captura la percepción de la medida en la que los agentes confían en las reglas de la sociedad y cumplen con ellas, y en particular la calidad de la ejecución de contratos, derechos de propiedad, la policía y los tribunales.

- Control de la corrupción: captura la percepción de la medida en que el poder público se ejerce en beneficio del sector privado, así como la "captura" del Estado por minorías selectas e intereses privados.

Estas variables fueron obtenidas de la base Worldwide Governance Indicators del Banco Mundial. Como se puede inferir de las definiciones de las variables, los indicadores se construyen empleando encuestas de opinión que capturan la percepción de los informantes. Las fuentes subyacentes de los indicadores incluyen: i) encuestas de hogares y firmas; ii) organismos no gubernamentales; iii) proveedores comerciales de información y iv) organizaciones del sector público.

Las fechas en las cuales los países incurrieron en una crisis de deuda soberana y los costos asociados a dichas crisis se obtuvieron de Laeven y Valencia (2013). Esta base de datos incluye a todas las crisis de deuda soberanas del periodo 1970-2011. Los autores fechan los episodios de los defaults y reestructuraciones soberana 
basándose en información de Beim y Calomiris (2001), Banco Mundial (2002), Sturzenegger y Zettelmeyer (2006), reportes del Staff del FMI y reportes de las agencias calificadoras de crédito. La información que compilan incluye el año en el que el soberano hizo default a los acreedores privados y el año en que reestructuró su deuda. Usando este enfoque, Laeven y Valencia (2013) identifican 66 episodios de crisis soberana y reestructuración durante el periodo 1970-2011.

El monto de deuda soberana en default en manos de acreedores privados se obtuvo de Beers y Nadeau (2014), quienes recopilan la primera base de datos que realiza dichas estimaciones desagregadas de acuerdo al tenedor del activo en el periodo 1975-2013, combinando múltiples bases de datos e incorporando información nueva. Los autores identifican a los episodios de default en los casos en que los servicios de la deuda no se hayan pagado en la fecha de vencimiento; no se hayan realizado los pagos dentro de las condiciones estipuladas si tenía garantía; o -en ausencia de un default común y corriente- en el caso de que, como consecuencia del accionar del soberano, los acreedores incurran en pérdidas económicas debido a su tenencia de deuda pública (cf. Beers y Nadeau, 2014, p.2). De esta base también se infiere la cantidad de años en default que pasaron los soberanos durante el periodo bajo análisis.

Finalmente, las quitas asociadas a cada reestructuración se obtuvieron de Cruces y Trebesch (2013). Combinando información de los archivos del FMI, investigaciones del sector privado, así como artículos de la prensa financiera, los autores construyen la primera base completa de reestructuraciones de deuda llevadas a cabo con bancos extranjeros y tenedores de bonos durante el periodo 1970-2010. La base de datos incluye estimaciones sobre los "recortes" asociados a 180 reestructuraciones llevadas a cabo por 68 país. 
Gráfico E.1 Nube de puntos y ajuste lineal de los spreads y percepción del mercado
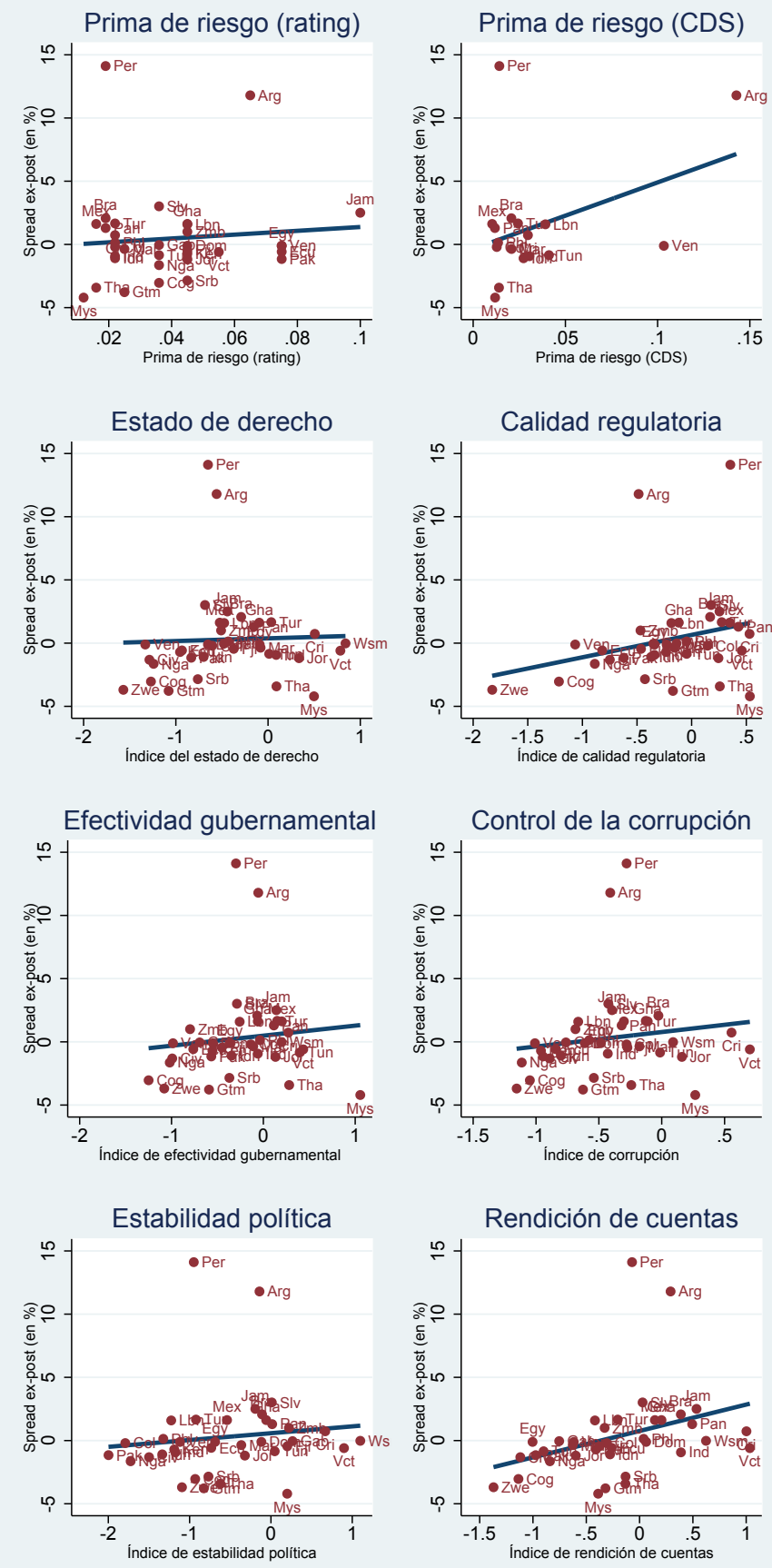
Gráfico E.2 Nube de puntos y ajuste lineal de los spreads y gestión de la deuda
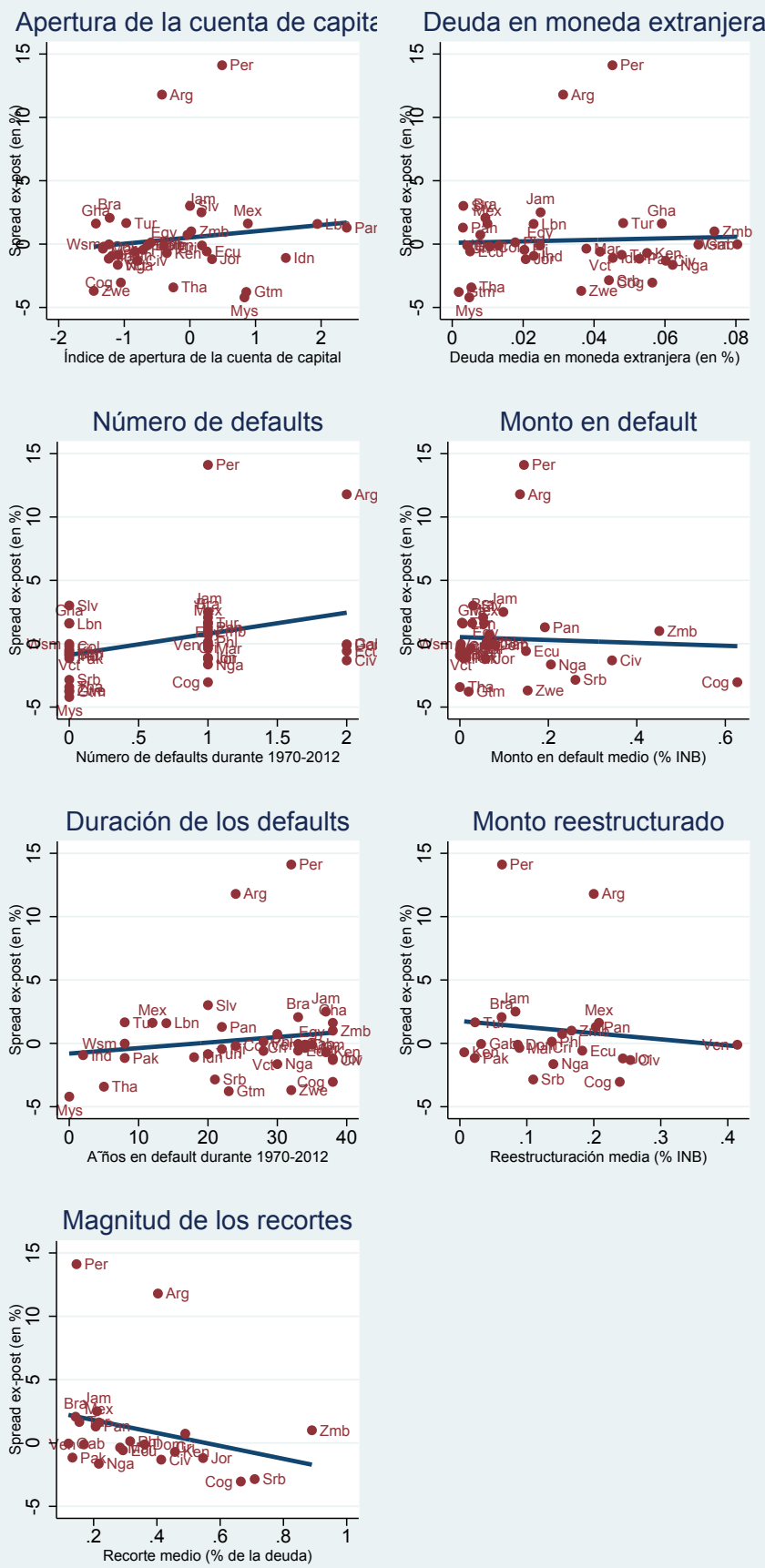


\section{Referencias}

Banco Mundial (2002). Global Development Finance, Apéndice sobre reestructuraciones de deuda comercial. Washington, DC: World Bank.

Beers, D. y J. S. Nadeau (2014). "Introducing a New Database of Sovereign Defaults", Bank of Canada, Technical Report No. 101.

Beim, D. y C. Calomiris (2001). Emerging Financial Markets, Apéndice del Capítulo 1. New York: McGraw-Hill/Irwin Publishers.

Benjamin, D. y M. Wright (2013). "Recovery Before Redemption: A Theory of Delays in Sovereign Debt Renegotiations", Centre for Applied Macroeconomic Analysis, Working Paper no. 15.

Bulow, J. y K. Rogoff (1988). "Sovereign Debt: Is To Forgive To Forget?", American Economic Review, vol. 79(1), pp. 43-50.

Chinn, M. y H. Ito (2006). "What Matters for Financial Development? Capital Controls, Institutions, and Interactions", Journal of Development Economics, vol. 81(1), pp. 163-192.

Cruces, J. J. y C. Trebesch (2013). "Sovereign Defaults: The Price of Haircuts", American Economic Journal: Macroeconomics, vol. 5(3), pp. 85-117.

Eaton, J. y M. Gersovitz (1981). "Debt with Potential Repudiation: Theoretical and Empirical Analysis", Review of Economic Studies, vol. 48(2), pp. 289-309.

Eichengreen, B. y R. Portes (1986). "Debt and Default in the 1930s: Causes and Consequences", European Economic Review, vol. 30, pp. 599-640.

Grossman, H. y J. B. Van Huyck (1988). "Sovereign Debt as a Contingent Claim: Excusable Default, Repudiation, and Reputation", American Economic Review, 
vol. $78(5)$, pp. 1088-1097.

Heymann, D. (2007). "Macroeconomía de las Promesas Rotas", Revista de economía política de Buenos Aires, vol. 2(1), pp. 27-41.

Klingen, C., B. Weder y J. Zettelmeyer (2004). "How Private Creditors Fared in Emerging Debt Markets, 1970-2000", Centre for Economic Policy Research, Discussion Paper No. 4374.

Laeven, L. y F. Valencia (2013). "Systemic Banking Crises Database: An Update", IMF Economic Review, vol. 61(2), pp. 225-270.

Lindert, P. (1989). "Response to Debt Crisis: What Is Different about the 1980s?", en The International Debt Crisis in Historical Perspective, ed. B. Eichengreen y P. Lindert (Cambridge, Mass. Y London: The MIT Press), pp. 227-75.

Lindert, P. y P. Morton (1989). "How Sovereign Debt Has Worked", en Developing Country Debt and Economic Performance, vol. 1, ed. J. Sachs (Chicago: The University of Chicago Press), pp. 39-105.

Reinhart, C. y K. Rogoff (2009). This Time Is Different: Eight Centuries of Financial Folly. Economics Books, Princeton University Press.

Rieffel, L. (2003). Restructuring Sovereign Debt. Washington D.C: Brookings Institution Press.

Sánchez, F. (1991). "Rentabilidad de la deuda externa de la Región Andina durante el siglo XIX, 1840-1914. Un análisis empírico", Cuadernos de Economía, vol. 11(16), pp. 186-204.

Suter, C. (1992). Debt Cycles in the World-Economy. Foreign Loans, Financial Crises, and Debt Settlements, 1820-1990. Bolder: Westview Press. 
Sturzenegger, F. y J. Zettelmeyer (2007). Debt defaults and lessons from a decade of crises. Cambridge, Mass: MIT Press.

Sturzenegger, F. y J. Zettelmeyer (2008). "Haircuts: Estimating investor losses in sovereign debt restructurings, 1998-2005", Journal of International Money and Finance, vol. 27(5), pp. 780-805.

Tomz, M. y M. Wright (2013). "Empirical Research on Sovereign Debt and Default", Annual Review of Economics, vol. 5(1), pp. 247-272.

Winkler, M. (1933). Foreign Bonds. An Autopsy: A Study of Defaults and Repudiations of Government Obligations. Philadelphia: Roland Swain.

Yue, V. (2010). "Sovereign default and debt renegotiation", Journal of International Economics, vol. 80(2), pp. 176-187. 